\title{
Performance and robustness of probabilistic river forecasts computed with quantile regression based on multiple independent variables
}

\author{
F. Hoss and P. S. Fischbeck \\ Carnegie Mellon University, Department of Engineering \& Public Policy, 5000 Forbes Avenue, Pittsburgh, PA 15213, USA \\ Correspondence to: F. Hoss (fraukehoss@gmail.com)
}

Received: 28 August 2014 - Published in Hydrol. Earth Syst. Sci. Discuss.: 14 October 2014

Revised: 28 July 2015 - Accepted: 27 August 2015 - Published: 25 September 2015

\begin{abstract}
This study applies quantile regression (QR) to predict exceedance probabilities of various water levels, including flood stages, with combinations of deterministic forecasts, past forecast errors and rates of water level rise as independent variables. A computationally cheap technique to estimate forecast uncertainty is valuable, because many national flood forecasting services, such as the National Weather Service (NWS), only publish deterministic singlevalued forecasts. The study uses data from the 82 river gauges, for which the NWS' North Central River Forecast Center issues forecasts daily. Archived forecasts for lead times of up to 6 days from 2001 to 2013 were analyzed. Besides the forecast itself, this study uses the rate of rise of the river stage in the last 24 and $48 \mathrm{~h}$ and the forecast error 24 and $48 \mathrm{~h}$ ago as predictors in QR configurations. When compared to just using the forecast as an independent variable, adding the latter four predictors significantly improved the forecasts, as measured by the Brier skill score and the continuous ranked probability score. Mainly, the resolution increases, as the forecast-only QR configuration already delivered high reliability. Combining the forecast with the other four predictors results in a much less favorable performance. Lastly, the forecast performance does not strongly depend on the size of the training data set but on the year, the river gauge, lead time and event threshold that are being forecast. We find that each event threshold requires a separate configuration or at least calibration.
\end{abstract}

\section{Introduction}

River-stage forecasts are no crystal ball; the future remains uncertain. The past has shown that unfortunate decisions have been made, because of users' unawareness of the magnitude of potential forecast errors (Pielke, 1999; Morss, 2010). For many users, such as emergency managers, forecasts are most important in extreme situations, such as droughts and floods. Unfortunately, it is exactly in those situations that forecasts are the most uncertain, i.e., forecast errors are the largest, due to the infrequency and the subsequent scarcity of data.

Currently, the National Weather Service (NWS) does not routinely publish uncertainty information along with their deterministic short-term river-stage forecast (Fig. 1). Given the many sources and complexity of uncertainty and the lacking user experience, it is easy to see how forecast users find it difficult to estimate the forecast error. Additionally, users might only experience such an event once or twice in their lifetime, so that they have no experience as to what extent they can rely on forecasts in such situations. Including uncertainty in river forecasts would therefore be valuable, just as has been recommended for weather forecasts in general (e.g., National Research Council, 2006). Hopefully, decisionmakers would then consider the whole bandwidth of possible future water levels, rather than focusing on the best estimate that is currently being published.

There are two types of approaches to estimate forecast uncertainty (e.g., Leahy, 2007; Demargne et al., 2013; Regonda et al., 2013): those addressing major sources of uncertainty individually, e.g., input uncertainty and hydrological uncertainty, and those taking into account all sources 


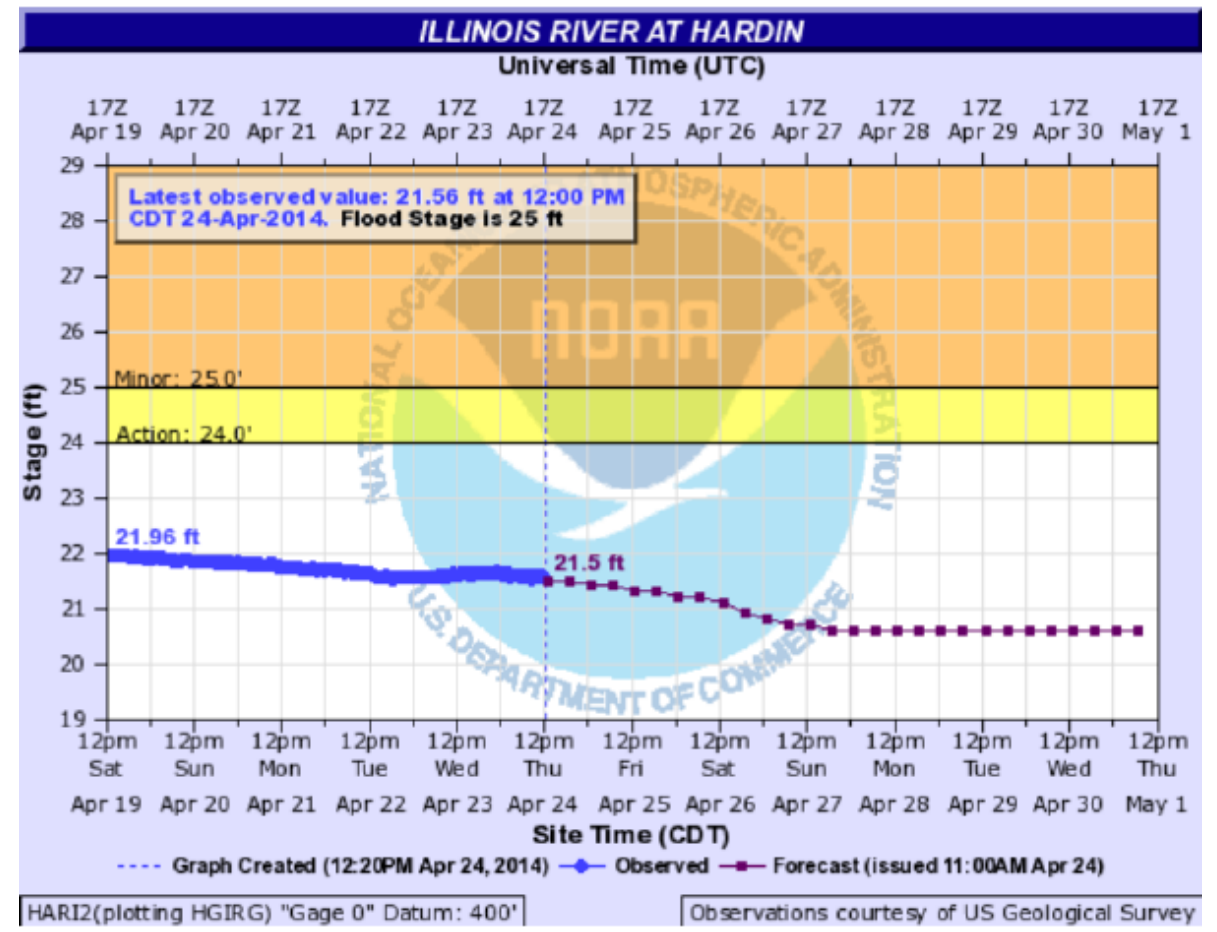

Figure 1. Deterministic short-term weather forecast in $6 \mathrm{~h}$ intervals as published by the NWS for Hardin, IL, on 24 April 2014 (source: http://water.weather.gov/ahps2/hydrograph.php?wfo=lsx\&gage=hari2).

of uncertainty in a lumped fashion. Both approaches have their advantages and disadvantages. When sources of uncertainty are modeled separately, their different characteristics can be taken into account (e.g., some sources of uncertainty depend on lead time, while others do not). Consequently, the approach addressing the major sources of output uncertainty is likely to result in better performing, more parsimonious model configurations. On the downside, this approach is expensive to develop, maintain and run. The alternative, i.e., the lumped quantification of uncertainties, is a less demanding in development and computation runtime but glosses over many of the finer details of uncertainties (Regonda et al., 2013).

Most previously developed post-processors to generate probabilistic forecasts share the overall setup but differ in their implementation. Independent variables such as the forecasted and observed river stage, river flow or precipitation, and previous forecast errors are used to predict the forecast error, conditional probability distribution of the forecast error or other measures of uncertainty for various lead times (e.g., Kelly and Krzysztofowicz, 1997; Montanari and Brath, 2004; Montanari and Grossi, 2008; Regonda et al., 2013; Seo et al., 2006; Solomatine and Shrestha, 2009; Weerts et al., 2011). These techniques differ in a number of ways, including their sub-setting of data and the output metric. Please see Regonda et al. (2013) and Solomatine and Shrestha (2009) for a summary of each technique.

The National Weather Service has chosen to quantify the most significant sources of uncertainty using ensemble tech- niques (Demargne et al., 2013). The NWS has developed the Hydrologic Ensemble Forecast Service (HEFS) to be able to provide short-term and medium-term probabilistic forecasts (Demargne et al., 2013). HEFS includes a post-processor, the Hydrologic Ensemble Post-Processor (EnsPost). It models the hydrological uncertainty by estimating the probability distribution for each of the ensemble members which have been produced with varying input to account for input uncertainty (NWS-OHD, 2013). The Experimental Ensemble Forecast Service (XEFS) additionally features the more parsimonious Hydrologic Model Output Statistics (HMOS) streamflow ensemble processor, which estimates the total uncertainty (input and hydrological uncertainty) of singlevalued streamflow forecasts based on conditional probability distributions (US Department of Commerce/NOAA, 2012).

This paper further develops one of the techniques mentioned above: the quantile regression approach to postprocess river forecasts first introduced by Wood et al. (2009) and further elaborated by Weerts et al. (2011) and López López et al. (2014). In a comparative analysis of four different post-processing techniques to generate confidence intervals, the quantile regression technique was one of the two most reliable techniques (Solomatine and Shrestha, 2009), while being the mathematically least complicated and requiring few assumptions. After Wood et al. (2009) presented the proof of concept for the Lewis River in Washington State at a conference, Weerts et al. (2011) published a formal study of quantile regression to compute confidence intervals for 
river-stage forecasts. Weerts et al. (2011) achieved impressive results in estimating the 50 and $90 \%$ confidence interval of river-stage forecasts for three case studies in England and Wales using QR (quantile regression) with calibration and validation data sets spanning 2 years each. When applying QR to river forecasts, Weerts et al. (2011) transformed the deterministic forecasts and the corresponding forecast errors into the Gaussian domain using normal quantile transformation (NQT) to account for heteroscedasticity. Building on the Weerts et al. (2011) study, López López et al. (2014) compare different configurations of QR with the forecast as the only independent variable, including configurations without NQT and preventing the crossing of quantiles. They found that no configuration was consistently superior for a range of forecast quality measures (López López et al., 2014).

This paper combines elements of the studies mentioned above. In some aspects, our approach differs from those three studies. We predict the exceedance probabilities of flood stages rather than uncertainty bounds. Additionally, we are fortunate to have a much larger data set than the three earlier studies, consisting of archived forecasts for 82 river gauges covering 11 years. Furthermore, we introduce additional predictors, as was suggested by López López et al. (2014). This study does not add to the mathematical technique of quantile regression itself.

The proposed QR approach is similar to the HMOS approach, but it differs in the following ways. First, HMOS uses ordinary linear regression instead of quantile regression. Second, the QR method uses the single-valued forecast, rates of rise and past forecast errors as independent variables, while HMOS includes recently observed and current flows and quantitative precipitation forecasts (QPFs) as predictors. Third, in this paper, QR models are built for a number of event thresholds, whereas HMOS develops models for subsets of forecasted streamflows (Regonda et al., 2013).

Identifying the best-performing set of independent variables is central to this paper. All possible combinations of the following predictors have been studied: forecast, the rate of rise of water levels in past hours, and the past forecast errors. Additionally, the robustness of the resulting QR configurations across different sizes of training data sets, locations, lead times, water levels, and forecast year has been assessed.

The paper is structured as follows. The Data section describes the used data and reviews the overall forecast error for the data set. The Method section introduces quantile regression and the performance measures, and discusses the performed analyses. The Results describes the results of identifying the best-performing set of independent variables. Additionally, it discusses the robustness of the studied QR configurations. The fourth and last section presents the conclusions and proposes further research ideas.

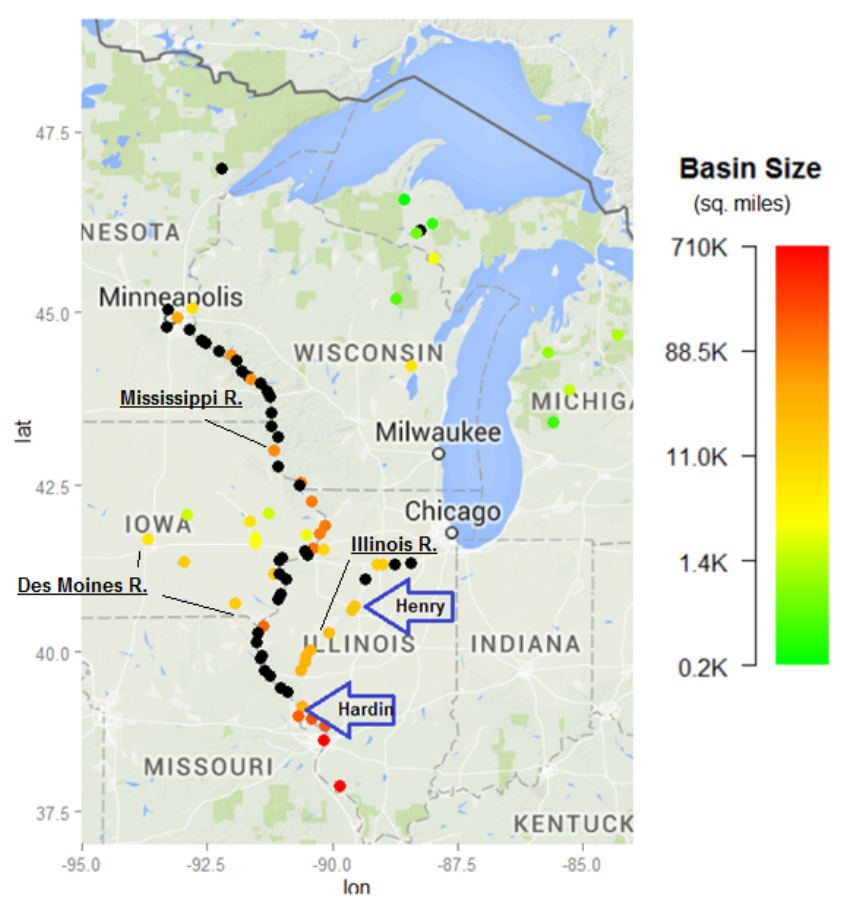

Figure 2. River gauges for which the North Central River Forecast Center publishes forecasts daily. Henry (HYNI2) and Hardin (HARI2) are indicated with arrows. For gauges indicated by black dots the basin size is missing. The color scale for basin size in square miles is logarithmic.

\section{Data}

The NWS's daily short-term river forecasts predict the stage height in $6 \mathrm{~h}$ intervals for up to 6 days ahead $(206 \mathrm{~h}$ intervals). When floods occur and more information is needed, the local river forecast center (RFC) can decide to publish river-stage forecasts more frequently and for more locations. Welles et al. (2007) provides a detailed description of the forecasting process.

For this paper, all forecasts published by the North Central River Forecast Center (NCRFC) between 1 May 2001 and 31 December 2013 were requested from the NCDC's HDSS Access System (National Climatic Data Center, 2014; Station ID: KMSR, Bulletin ID: FGUS5). In total, the NCRFC produces forecasts for 525 gauges. For 82 of those gauges, forecasts have been published daily for at least 2 years, and are not inflow forecasts. The latter have been excluded from the forecast error analysis because they forecast discharge rather than water level. About half of the analyzed gauges are along the Mississippi River (Fig. 2). The Illinois River and the Des Moines River are two other prominent rivers in the region. The drainage areas of the 82 river gauges average $61500 \mathrm{mi}^{2}$ (minimum $200 \mathrm{mi}^{2}$; maximum $708600 \mathrm{mi}^{2}$ ). Figure 3 shows an empirical cumulative density function of drainage areas sizes. 


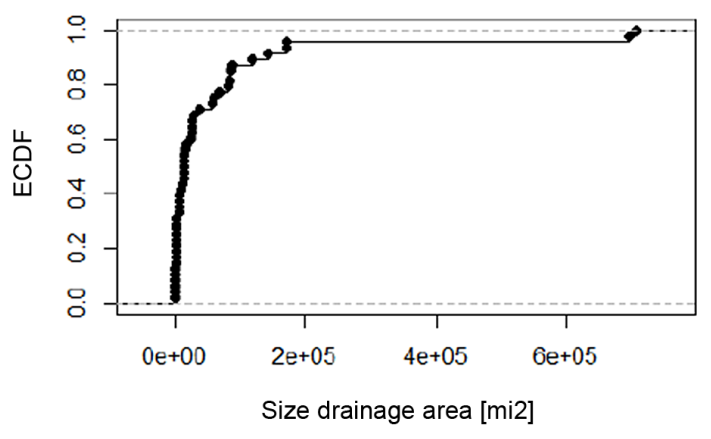

Figure 3. Empirical cumulative density function (ecdf) of sizes of drainage area for the river gauges that are being forecasted daily by the NCRFC.

Two river gauges serve as an illustration for the points made throughout this paper. These two gauges were chosen to capture different but representative conditions. Hardin, IL, is just upstream of the confluence of the Illinois River and the Mississippi River (Fig. 2). Therefore, it can experience backwatering, when the high water levels in the Mississippi River prevent the Illinois River from draining. Henry, IL, is located $\sim 200$ mi upstream of Hardin, having a difference in elevation of $\sim 25 \mathrm{ft}$. The Illinois River is $\sim 330 \mathrm{mi}$ long (Illinois Department of Natural Resources, 2011), draining an area of $\sim 13500 \mathrm{mi}^{2}$ at Henry (USGS, 2015a) and $\sim 28700 \mathrm{mi}^{2}$ at Hardin (USGS, 2015b). The number of case studies has been limited to two because of computation time.

In general, the NCRFC's forecasts are well calibrated across the entire data set. The average error, defined as observation minus the forecast, is zero for most gauges (Fig. 4). For lead times longer than 3 days, a slight underestimation by the forecast is noticeable. By a lead time of 6 days this underestimation averages $0.41 \mathrm{ft}$ (Figs. 4a, 5). Extremely low water levels, defined as below the 10th percentile of observed water levels, are also well calibrated (Figs. 4b, 5). However, when considering higher water levels the picture changes. When only observations exceeding the 90th percentile of all observations are considered, the underestimation becomes more pronounced, averaging $0.29 \mathrm{ft}$ for 3 days of lead time and $1.14 \mathrm{ft}$ for 6 days of lead time (Figs. 4c, 5). When only looking at observations that exceeded the minor flood stages corresponding to each gauge, the underestimation averages $0.45 \mathrm{ft}$ for 3 days of lead time and $1.51 \mathrm{ft}$ for 6 days of lead time (Figs. 4d, 5). However, some gauges, such as Morris (MORI2), Marseilles Lock/Dam (MMOI2) - both on the Illinois River - and Marshall Town on the Iowa River (MIWI4) experience average errors of 5-12 ft for water levels higher than the minor flood stage. The gauges MORI2 and MMOI2 are upstream of a dam. It is possible that the forecasts performed so poorly there because the dam operators deviated from the schedules that they provide the river forecast centers to base their calculations on. In sum, predicting the forecast error distribution as is done in this paper has much added value for forecast users, because the forecast error can amount to several feet.

\section{Method}

QR is used to estimate the distribution of river-stage forecasts for each forecast point in time and location. This information can be published in a number of formats to suit the needs of the forecast users. Wood et al. (2009) and Weerts et al. (2011) chose to study confidence intervals. A confidence interval is the range between two points on the estimated forecast distribution, e.g., between the 10th and 90th percentiles. Our paper differs in that our output is the probability of exceeding a flood stage. A flood stage and the corresponding probability of it being exceeded are represented by a single point on the estimated forecast distribution. Assessing forecast performance for a single point rather than for two points on the estimated distribution allows for scrutinizing forecast performance more closely, not least because the method is not necessarily equally successful in both tails of the distribution.

In the following, quantile regression itself and the analysis to identify the best-performing set of independent variables are explained.

\subsection{Quantile regression}

In the context of river forecasts, linear quantile regression has been used to estimate the distribution of forecast errors as a function of the forecast itself. Weerts et al. (2011) summarize this stochastic approach as follows: "[It] estimates effective uncertainty due to all uncertainty sources. The approach is implemented as a post-processor on a deterministic forecast. [It] estimates the probability distribution of the forecast error at different lead times, by conditioning the forecast error on the predicted value itself. Once this distribution is known, it can be efficiently imposed on forecast values."

Quantile regression was first introduced by Koenker (2005) and Koenker and Bassett (1978). It is different from ordinary least square regression in that it predicts percentiles rather than the mean of a data set. Koenker and Machado (1999, p. 1305) and Alexander et al. (2011) demonstrate that studying the coefficients and their uncertainty for different percentiles generates new insights, especially for non-normally distributed data.

López López et al. (2014) did not find that the quantile regression method produces better forecasts if the variables are subject to NQT beforehand, as was practiced by Weerts et al. (2011). We chose not to apply NQT, because four of five of our independent variables are already approximately normally distributed, only the forecast itself is not.

A quantile regression is run for each lead time and desired percentile with the forecast error as the dependent variable and the forecast and other variables as independent variables. To prevent the quantile regression lines from crossing each 

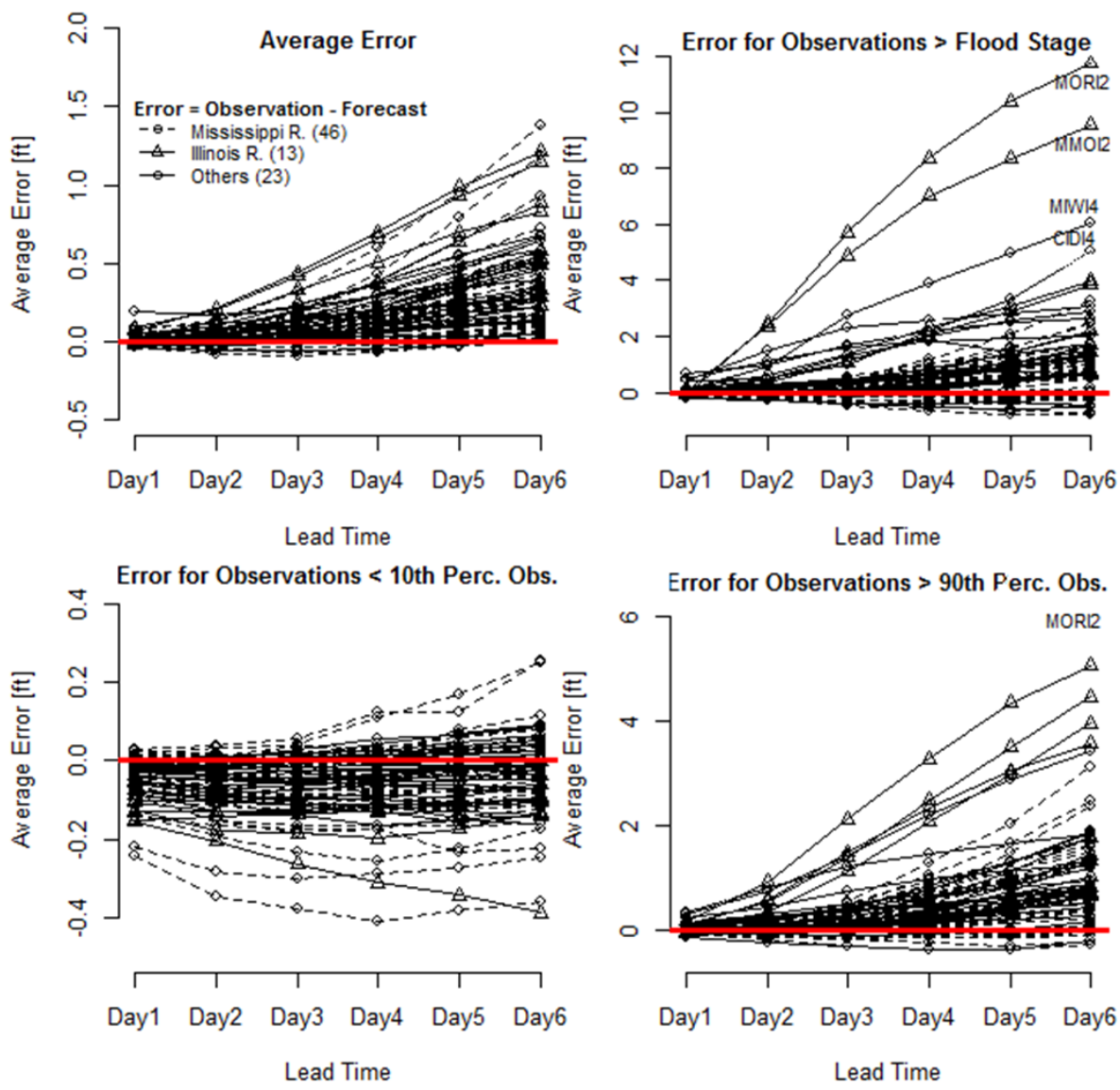

Figure 4. Forecast error for 82 river gauges that the NCRFC publishes daily forecasts for. In counterclockwise direction starting at the top left: (a) average error; (b) error on days that the water level did not exceed the 10th percentile of observations; (c) error on days that the water level exceeded the 90th percentile of observations; (d) error on days that the water level exceeded minor flood stage.

other, a fixed-effects model is implemented below a certain forecast value. Weerts et al. (2011) give a detailed mathematical description for applying QR to river forecasts. Detailed instructions to perform NQT can be found in Bogner et al. (2012). Mathematically, the approach is formulated as follows (with and without NQT).

Equation (1): QR configuration with NQT, with percentiles of the forecast error as the dependent variable and the one independent variable, both transformed into the normal domain.

$F_{\tau}(t)=\operatorname{fcst}(t)+\mathrm{NQT}^{-1}\left[\sum_{i}^{I} a_{i, \tau} \cdot V_{\mathrm{NQT}, i}(t)+b_{\tau}\right]$

Equation (2): QR configuration without NQT, with percentiles of the forecast error as the dependent variable and multiple independent variables.

$F_{\tau}(t)=\operatorname{fcst}(t)+\sum_{i}^{I} a_{i, \tau} \cdot V_{i}(t)+b_{\tau}$

where $F_{\tau}(t)$ is the estimated forecast associated with percentile $\tau$ and time $t, f \operatorname{cst}(t)$ is the original forecast at time $t$,
$V_{i}(t)$ is the independent variable $i$ (e.g., the original forecast) at time $t, V_{i ; \mathrm{NQT}}(t)$ is the independent variable $I$ transformed by NQT at time $t$ and $a_{i, \tau}$ and $b_{\tau}$ are configuration coefficients.

The second part of the equations stands for the error estimate based on the quantile regression configuration for each error percentile $\tau$ and lead time. In Eq. (1), used by Weerts et al. (2011), this estimation was executed in the Gaussian domain using only the forecast as independent variable. Our study mainly uses Eq. (2), i.e., it does not transform the predictors and the predictand. All quantile regressions were done using the command $\mathrm{rq}()$ in the R package "quantreg" (Koenker, 2013).

\subsection{Verification measures}

The QR configuration by Weerts et al. (2011) was evaluated by determining the fraction of observations that fell into the confidence intervals predicted by the $\mathrm{QR}$ configuration; i.e., ideally, $80 \%$ of the observations should be larger than the predicted 10th percentile for that day, and smaller than the predicted 90th percentile. López López et al. (2014) used 

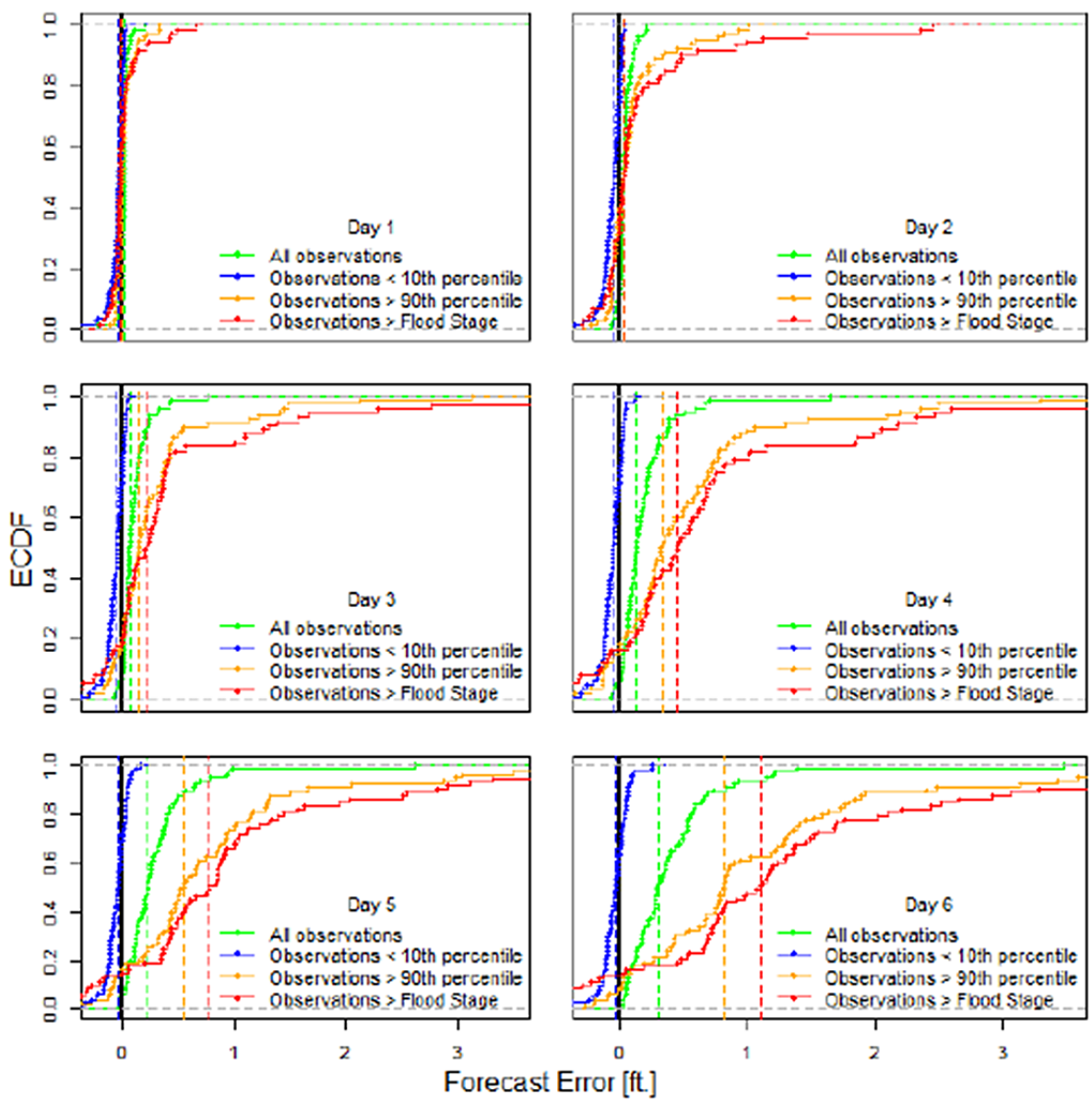

Figure 5. Empirical cumulative distribution function (ecdf) of forecast error at 82 river gauges for six lead times. Vertical lines show the median forecast error of the corresponding subset.

a number of measures to assess configuration performance, e.g., the Brier skill score (BSS), the mean continuous ranked probability (skill) score (CRPSS), the relative operating characteristic (ROC), and reliability diagrams to compare QR configurations.

We focus on the BSS - first introduced by Brier (1950) - to assess QR configurations for three reasons. First, to be able to determine the best set of predictors it is easiest to choose a single measure. Second, the BSS allows us to study forecast performance at individual event thresholds. Third, out of the available measures, the Brier score is attractive because it can be decomposed into two different measures of forecast quality (see Eq. 3): reliability and resolution. The third component is uncertainty. This type of uncertainty describes the uncertainty inherent in an event caused by natural variability. It is narrower than forecast uncertainty, because the latter additionally includes the uncertainty that is caused by imperfections of the forecast model, i.e., the variables that could explain some of the uncertainty have not been identified or correctly parameterized yet. In sum, the BS' uncertainty term is not subject to the forecast quality. Equation 3 gives the definition of the (decomposed) Brier score (e.g., Jolliffe and Stephenson, 2012; Wikipedia, 2014; WWRP/WGNE, 2009).

Equation (3): Brier score decomposed into three terms: reliability, resolution and uncertainty.

$$
\begin{aligned}
\mathrm{BS} & =\underbrace{\frac{1}{N} \sum_{k=1}^{K} n_{k}\left(f_{k}-\bar{o}_{k}\right)^{2}-\frac{1}{N} \sum_{k=1}^{K} n_{k}\left(\bar{o}_{k}-\bar{o}\right)^{2}+\underbrace{\bar{o}(1-\bar{o})}_{\text {Resolution }}}_{\text {Reliability }} \\
& =\frac{1}{N} \sum_{t=1}^{T}\left(f_{t}-o_{t}\right)^{2}
\end{aligned}
$$

where BS is the Brier score, $N$ is the number of forecasts, $K$ is the the number of bins for forecast probability of binary event occurring on each day, $n_{k}$ is the the number of forecasts falling into each bin, $\delta_{k}$ is the the frequency of binary event occurring on days in which forecast falls into bin $k, f_{k}$ is the forecast probability, $\delta$ is the frequency of binary event occurring, $f_{t}$ is the forecast probability at time $t$, and $o_{t}$ is the observed event at time $t$ (binary: 0 - event did not happen, 1 - event happened). 

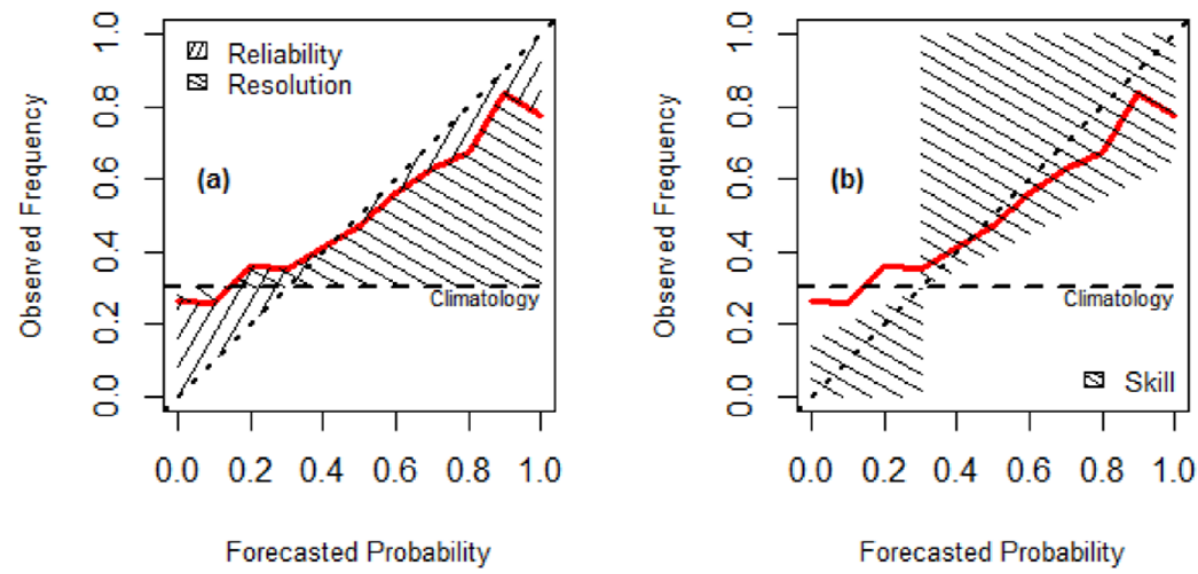

Figure 6. Theory behind Brier skill score illustrated for an imaginary forecast (red line): (a) reliability and resolution, (b) skill. In (a), the area representing reliability should be as small as possible and for resolution as large as possible. The forecast has skill (BSS $>0$ ), i.e., performs better than the reference forecast, if it is inside the shaded area in $(\mathbf{b})$. Ideally, the forecast would follow the diagonal $(\mathrm{BSS}=1)$ (adapted from Hsu and Murphy, 1986; Wilson, 2014).

The Brier score pertains to binary events, e.g., the exceedance of a certain river stage or flood stage. Reliability compares the estimated probability of such an event with its actual frequency. For example, perfect reliability means that on $60 \%$ of all days for which it was predicted that the water level would exceed flood stage with a $60 \%$ probability, it actually does so. The reliability curve for the forecast representing perfect reliability would follow the diagonal in Fig. 6, i.e., the area in Fig. 6a representing reliability would equal zero (Jolliffe and Stephenson, 2012; Wikipedia, 2014; WWRP/WGNE, 2009).

Resolution measures the difference between the predicted probability of an event on a given day and the historically observed average probability. For example, imagine a gauge where flood stage has historically been exceeded on $5 \%$ of the days in a year. If every day at that gauge the probability of exceeding flood stage is forecasted to be $5 \%$, the resolution of those forecasts would be zero. After all, the difference between the predicted frequency and the historical average is zero. So a forecast with higher resolution is better (e.g., Jolliffe and Stephenson, 2012; Wikipedia, 2014; WWRP/WGNE, 2009). In Fig. 6, the curve for a forecast with good resolution would be steeper than the dashed line that represents the historically observed frequency (climatology). It follows that forecasters should strive to maximize the area in Fig. 6a representing resolution. In absolute terms, the resolution can never exceed the uncertainty inherent to the river gauge, as represented by the third term in Eq. (3). (e.g., Jolliffe and Stephenson, 2012; Wikipedia, 2014; WWRP/WGNE, 2009).

A forecast performs better than the reference forecast (in this case the historically observed frequency), if it (the red line) is inside the shaded area in Fig. 6b. Then the forecast is said to have "skill". The BSS equals the Brier Score nor- malized by the historically observed frequency, i.e., the resolution and reliability terms are being divided by the uncertainty term (Eq. 4). In contrast to the Brier score, this makes the Brier skill score comparable across gauges with different frequencies of a binary event. The BSS can range from minus infinity to one. A BSS below zero indicates no skill; the perfect score is one (e.g., Jolliffe and Stephenson, 2012; Wikipedia, 2014; WWRP/WGNE, 2009).

Equation (4) shows the decomposition of the Brier skill score:

$\mathrm{BSS}=1-\frac{\mathrm{BS}}{\bar{o}(1-\bar{o})}=\frac{\mathrm{Res}}{\bar{o}(1-\bar{o})}-\frac{\mathrm{Rel}}{\bar{o}(1-\bar{o})}$,

where BSS is the Brier skill score, BS is the Brier score, Res is the resolution, Rel is the Reliability, $\bar{\delta}$ is the frequency of binary event occurring and $\bar{\delta}(1-\delta$ the climatological variance.

To verify that the results hold up for verification measures other than the BSS, we additionally use the Continuous Ranked Probability Score (CRPS). The BSS assesses forecast performance for one point on the forecast distribution, i.e., one event threshold. In contrast, the CRPS, defined by Eq. (5), measures the forecast performance for the forecast distribution as the whole. Therefore, the CRPS cannot detect whether the forecast does better or worse in the tails. Instead, it is a measure of the forecast's overall performance. The CRPS' perfect score equals zero (e.g., Jolliffe and Stephenson, 2012; WWRP/WGNE, 2009).

All measures of forecast quality were computed using the R package "verification" (NCAR, 2014).

CRPS $=\frac{1}{N} \sum_{n=1}^{N} \int_{-\infty}^{\infty}\left(F_{n}^{\mathrm{f}}(x)-F_{n}^{\mathrm{o}}(x)\right)^{2} \mathrm{~d} x$ 
where CRPS is the continuous ranked probability score, $F_{n}^{\mathrm{f}}$ is the forecast probability distribution (cdf) for the $n$th forecast case, $F_{n}^{\mathrm{o}}$ is the observation for $n$th forecast case (cdf) and $N$ is the number of forecast cases, i.e., length of time series.

\subsection{Choice of independent variables}

The challenge is to identify a well-performing QR model with a set of predictors that is both parsimonious and comprehensive. Wood et al. (2009) found rate of rise and lead time to be informative independent variables. Weerts et al. (2011) achieved good results using only the forecast itself as predictor. Besides these variables, the most obvious predictors to include are the current water levels and those observed 24 and $48 \mathrm{~h}$ ago, and the forecast error 24 and $48 \mathrm{~h}$ ago (i.e., the difference between the current water level at issue time of the forecast that the error distribution is being predicted for and the forecasts that were produced 24 and $48 \mathrm{~h}$ earlier to predict the current water level). Additional potential independent variables are the water levels observed at gauges upand downstream at various times, the precipitation upstream of the catchment area, and the precipitation forecast.

Rates of rise and forecast errors were chosen to complement the forecast as independent variables. Therefore, instead of using it as an independent variable, separate QR models have been built for each lead time. After all, the best choice of independent variables might depend on lead time. Precipitation and precipitation forecast were not available for this study, because without direct access to the database at the NCDC requesting that data is a very lengthy effort.

Forecasts and observed water levels were readily accessible from NCDC databases. Rates of rise and forecast errors can be derived from those two. As will be shown in Sect. 4.3 , it is mathematically challenging to combine independent variables with different distributions into a joint predictor. Forecast and observed water levels have a skewed distribution, because low water levels occur more frequently than extremely high water levels, while rates of rise and forecast errors are approximately normally distributed. Accordingly, either forecasts and observations can easily be combined into a joint predictor or rates of rise and forecast errors. For this study the latter option was chosen for the following reasons. Observed water levels are systematically included in the NWS forecast model. Assuming a well-defined NWS forecast model, there should be no statistical relationship between forecast error and observed water levels. In comparison, rates of rise and forecast error are only included in the NWS model at the discretion of the individual forecaster. Therefore, the latter two variables are likely to contribute more information to predicting the distribution of forecast errors than the forecasts and observed water levels. Nonetheless, forecasts were included as the predictor in this study to demonstrate the difficulty of combining variables with a skewed distribution with normally distributed variables into a joint predictor, and because it served as the only indepen- dent variable in previous studies (Weerts et al., 2011; López López et al., 2014).

To determine which set of predictors performs best at generating probabilistic forecasts, all 31 possible combinations of the forecast (fcst), the rate of rise in the last 24 and $48 \mathrm{~h}$ (rr24, rr48), and the forecast error 24 and $48 \mathrm{~h}$ ago (err24, err48) - see Eq. (5) - were tested for 82 gauges that the NCRFC issues forecasts for every morning (Table 1). Based on the Bier skill score, it was determined which joint predictor delivers on average the best out-of-sample forecast performance for various lead times and water levels.

Equation (6) shows the QR configuration without NQT, with percentiles of the forecast error as the dependent variable and varying combinations of the five independent variables. This equation was used to predict the water level distribution for each day at 82 gauges with different lead times.

$$
\begin{array}{r}
F_{\tau}(t)=\mathrm{fcst}(t)+a_{\mathrm{fcst}, \tau} \cdot \mathrm{fcst}(t)+a_{\mathrm{rr} 24, \tau} \cdot \operatorname{rr} 24(t)+a_{\mathrm{rr} 48, \tau} \\
\cdot \operatorname{rr} 48(t)+a_{\mathrm{err} 24, \tau} \cdot \operatorname{err} 24(t)+a_{\mathrm{err} 48, \tau} \cdot \operatorname{err} 48(t)+b_{\tau}
\end{array}
$$

where $F_{\tau}(t)$ is the estimated forecast associated with percentile $\tau$ and time $t, f \operatorname{cst}(t)$ is the original forecast at time $t$, $\operatorname{rr} 24(t)$ and $\operatorname{rr} 48(t)$ are the rates of rise in the last 24 and $48 \mathrm{~h}$ at time $t, \operatorname{err} 24(t)$ and err48(t) are the forecast errors 24 and $48 \mathrm{~h}$ ago (e.g., the original forecast) at time $t$, and $a_{x x, \tau}$ and $b_{\tau}$ are the configuration coefficients, forced to be zero if the predictor is excluded from the joint predictor that is being studied.

\subsection{Computational process}

The final output of the computational process is the probability that a certain water level in the river or flood stage is exceeded on a given day, e.g., "on the day after tomorrow, the probability that the river exceeds $15 \mathrm{ft}$ at location X is $60 \%$." This is done in two steps. First, a training data set (first half of the data) is used to define one quantile regression configuration for each percentile of the error distribution $\pi=[0.05$, $0.1,0.15, \ldots, 0.85,0.90,0.95]$ and each lead time. The dependent variable is the forecast error, i.e., the difference between forecast and observed water level. To recap, depending on configuration (Table 1), the forecast itself, the rates of rise and forecast errors serve as independent variables.

In the second step, these QR configurations are used to predict percentile by percentile the distribution of forecast errors for each day in the verification data set (the second half of the data set). Effectively, for each day in the verification data set, a discrete probability distribution of forecast errors is predicted. Adding the single-valued forecast to the forecast error distribution results in a distribution of predicted water levels. Each estimated percentile $\pi$ contributes one point to that distribution.

Then, we calculate the probability with which various water levels (called event thresholds hereafter) will be exceeded. The probability of exceeding each water level is computed by 
Table 1. Joint predictors.

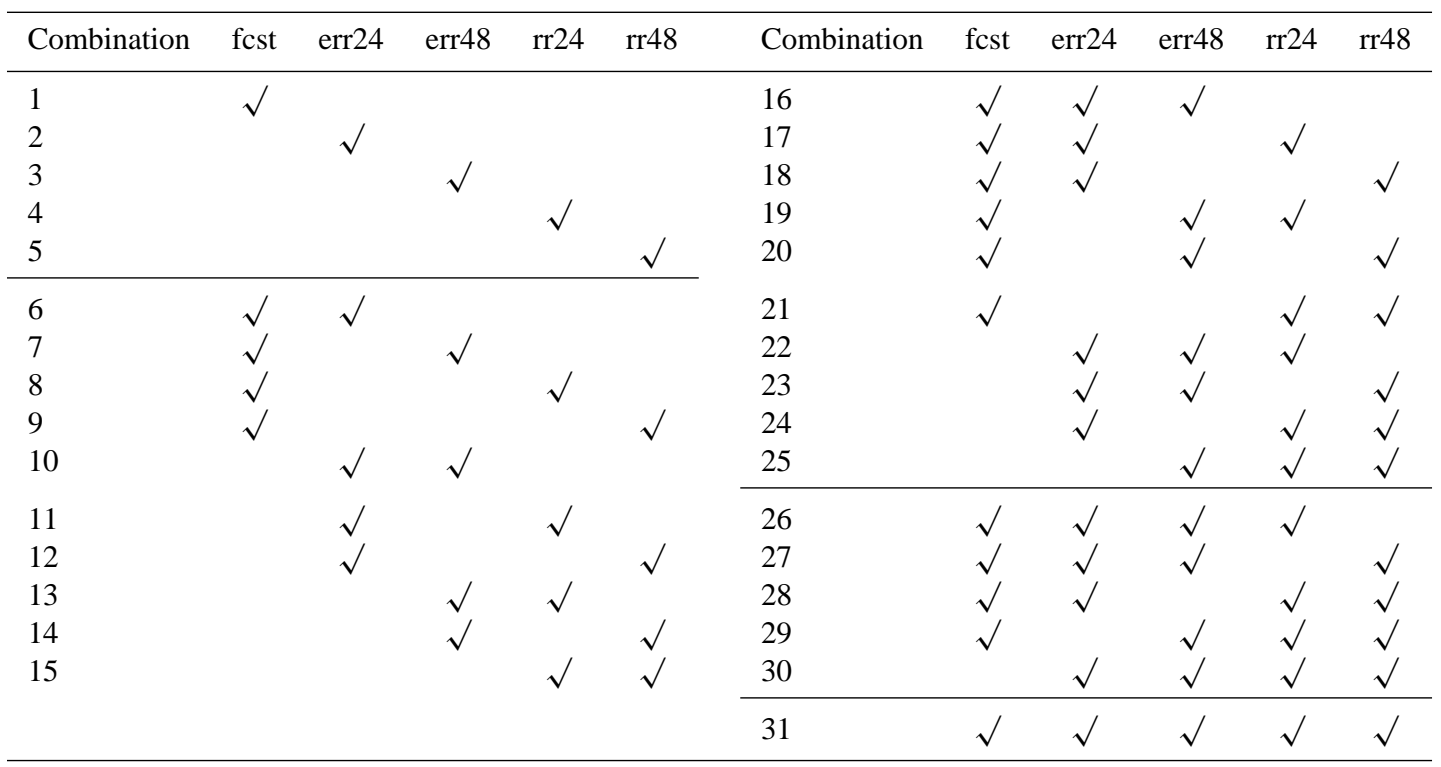

$\mathrm{fcst}=$ forecast; $\mathrm{rr} 24$ and $\mathrm{rr} 48=$ rise rate in the past 24 and $48 \mathrm{~h}$; err 24 and err $48=$ forecast error 24 and $48 \mathrm{~h}$ ago.

linearly interpolating between the points of the discrete probability distribution that was computed in the previous step. Next, the Brier skill score is determined based on predicted exceedance probability for all days in the verification data set.

To study whether the various combinations of predictors perform equally well for high and low thresholds, these last computational steps (i.e., interpolating to determine the exceedance probability for a certain water level and calculating the BSS) were repeated for eight event thresholds: the 10th, 25th , 75th, and 90th percentiles of observed water levels and the four decision-relevant flood stages (action stage, and minor, moderate, and major flood stage) of each gauge. Flood stages indicated when material damage or substantial hinder is caused by high water levels. Therefore, the flood stages correspond with different percentiles at different river gauges.

To determine the best-performing set of independent variables, the entire procedure is repeated for each of the 31 joint predictors in Table 1, thus using a different set of independent variables each time. The robustness of the technique was tested by analyzing its performance for 82 gauge locations using different lengths of data sets for five different lead times.

\section{Results}

In total, the BSS for 31 joint predictors (Table 1) across various lead times and event threshold have been compared. Across 82 river gauges, it has been analyzed which joint predictor delivers the best BSSs on average. When informative, the CRPS has been used as an additional measure of forecast performance.

\subsection{Identifying best-performing joint predictors on average}

For each river gauge, the combinations have been ranked by BSSs. The best-performing combination was ranked as first, the worst-performing as 31 st. It was found that the more independent variables are included in a joint predictor, the higher that set of predictors will rank on average (Fig. 7, Table 2a). Apparently, every additional independent variable does add information. In other words, the future forecast error is a function of rates of rise and past forecast errors. Rising water levels are difficult to anticipate and therefore a common source of forecast error, because precipitation is a major source of input uncertainty. For example, it is never completely certain into which river basin the rain will fall. Additionally, only the expected precipitation for the coming $12 \mathrm{~h}$ is currently included in forecasts, regardless of lead time. The past forecast errors are a measure of the magnitude of impact those unanticipated developments are likely to have.

For extremely high water levels, this trend favoring larger joint predictors gradually reverses (Fig. 8). The trend remains statistically significant, but its coefficient decreases for higher event thresholds (Table 2a) until it changes signs for major flood stages (Table 2b). A possible explanation is that combinations with more variables suffer from overfitting for extreme event thresholds characterized by data scarcity. 

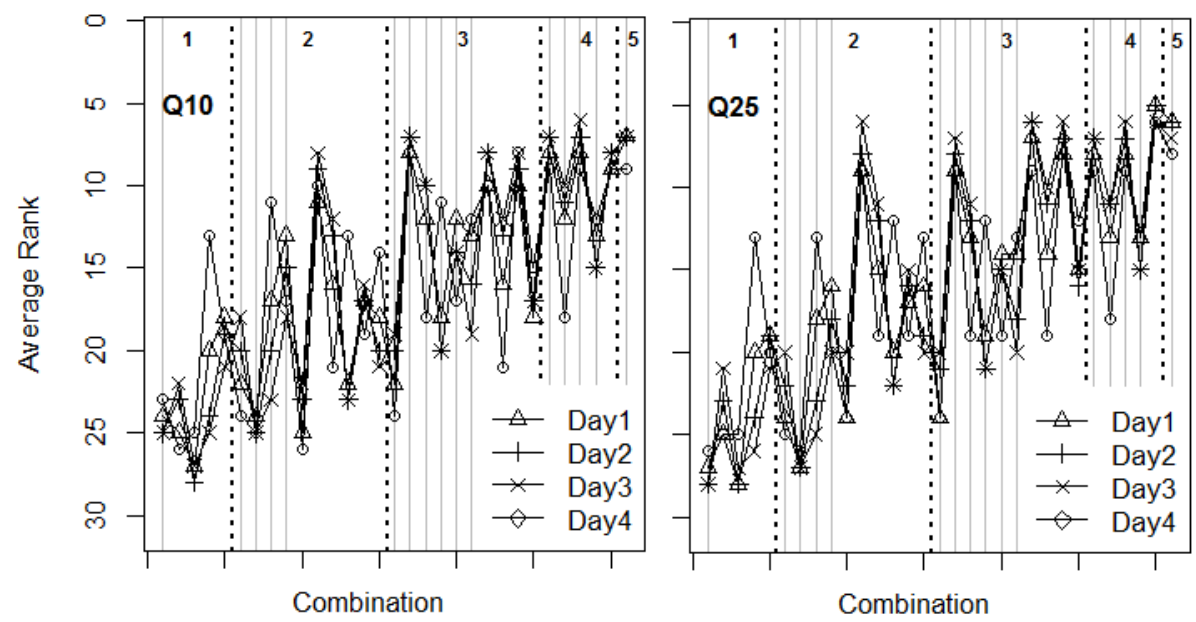

Figure 7. Average rank for each joint predictor for 1-4 days of lead time and two percentiles of observed water levels. Vertical gray lines correspond to the configurations that include the forecast as one of the predictors. The $y$ axis is reversed so that an increasing trend indicates increasing performance.
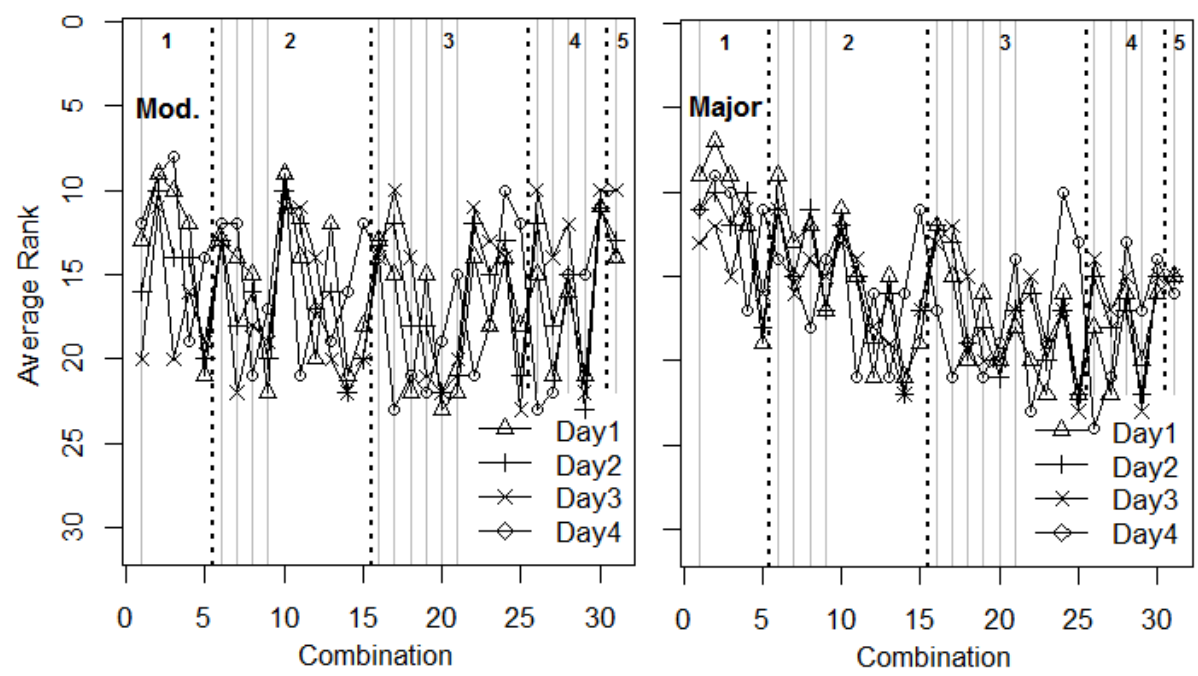

Figure 8. Average rank for each joint predictor for 1-4 days of lead time and the two highest flood stages. Vertical gray lines correspond to the configurations that include the forecast as one of the predictors. The $y$ axis is reversed so that an increasing trend indicates increasing performance.

The results hold up when CRPS instead of BSS is used as a measure of forecast performance. The average rank of joint predictors based on CRPS is proportional to the average rank as measured by the BSS previously (Fig. 9). However, scores themselves are not proportional (Fig. 10), because the BSS assesses one point on the estimated distribution, while the CRPS measures the forecast performance for the distribution as a whole. Figure 10 shows that BSS and CRPs correspond well for event thresholds $Q_{25}$ and $Q_{75}$. However, the BSS indicates that in the tails $\left(Q_{10}, Q_{90}\right)$ the forecast does not perform as well, i.e., despite equally good CRPS scores the BSS varies widely.

\subsection{Combining differently distributed variables into a joint predictor}

The combinations including the forecast (indicated by gray vertical lines in Figs. 7 and 8) perform significantly better than those that exclude it (Table 2). This disadvantageous impact of the forecast as an independent variable is less pronounced for very high or low event thresholds (Table 2a). Including the forecast into the joint predictor is even beneficial for major flood stages (Table $2 b$ ), when joint predictors with less rather than more variables perform better.

The forecast is difficult to combine with the other four predictors (err24/48, rr24/48) because their statistical distri- 
Table 2. Results of regression analyses to determine the impact of including more variables and the forecast into the joint predictor.

\begin{tabular}{lllll}
\hline \multicolumn{5}{l}{ (a) Percentiles of observed water levels } \\
\hline Independent variable: & $Q_{10}$ & $Q_{25}$ & $Q_{50}$ & $Q_{75}$ \\
Rank (1-31) & Coef. (St. Err.) & Coef. (St. Err.) & Coef. (St. Err.) & Coef. (St. Err.) \\
\hline Intercept & $26.49(.21)^{* * *}$ & $27.54(.19)^{* * *}$ & $24.47(.19)^{* * *}$ & $20.09(.22)^{* * *}$ \\
Number of variables & $-4.47(.08)^{* * *}$ & $-5.59(.08)^{* * *}$ & $-4.98(.08)^{* * *}$ & $-3.02(.09)^{* * *}$ \\
Forecast included? (binary) & $2.01(.17)^{* * *}$ & $5.15(.16)^{* * *}$ & $8.51(.16)^{* * *}$ & $7.18(.18)^{* * *}$ \\
$R^{2}$ & 0.23 & 0.34 & 0.33 & 0.17 \\
Adjusted $R^{2}$ & 0.23 & 0.34 & 0.33 & 0.17 \\
\hline & \multicolumn{5}{c}{ (b) Flood stages } & & \\
\hline Independent variable & Action FS & Minor FS & Moderate FS & Major FS \\
Rank (1-31) & Coef. (St. Err.) & Coef. (St. Err.) & Coef. (St. Err.) & Coef. (St. Err.) \\
\hline Intercept & $20.92(.22)^{* * *}$ & $18.76(.23)^{* * *}$ & $15.49(.27)^{* * *}$ & $12.58(.29)^{* * *}$ \\
Number of variables & $-3.33(.09)^{* * *}$ & $-2.40(.09)^{* * *}$ & $-0.22(.11)^{*}$ & $1.59(-12)^{* * *}$ \\
Forecast included? (binary) & $7.11(.18)^{* * *}$ & $6.68(.19)^{* * *}$ & $2.02(.22)^{* * *}$ & $-1.30(.24)^{* * *}$ \\
$R^{2}$ & 0.18 & 0.13 & 0.01 & 0.03 \\
Adjusted $R^{2}$ & 0.18 & 0.13 & 0.01 & 0.03 \\
\hline
\end{tabular}

$p$ values: ***: $<0.001 ; * *: 0.01 ; *: 0.05 ;():$.0.1 .
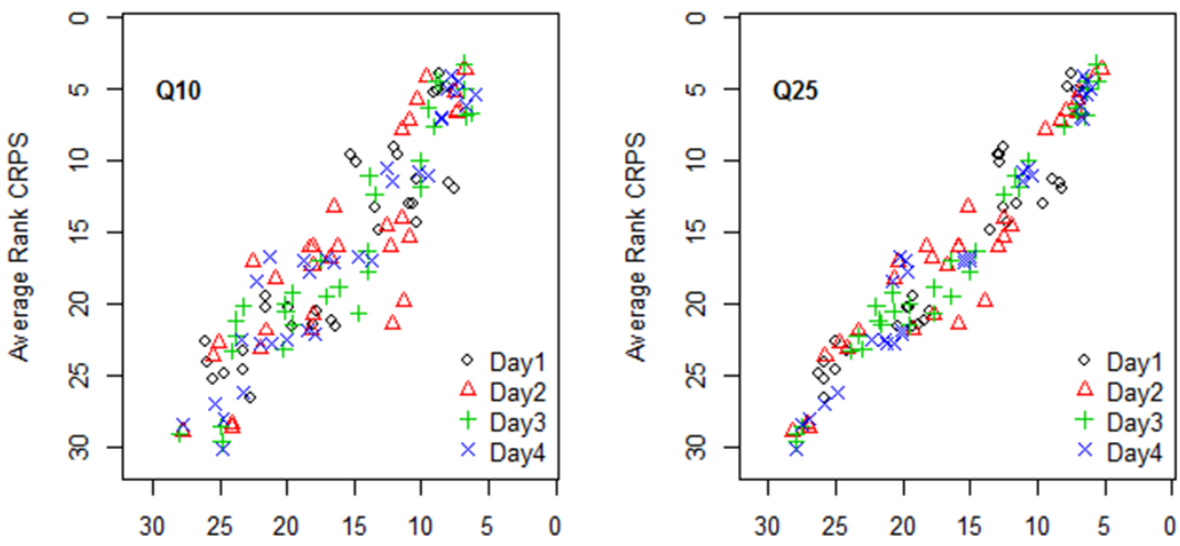

Average Rank BSS
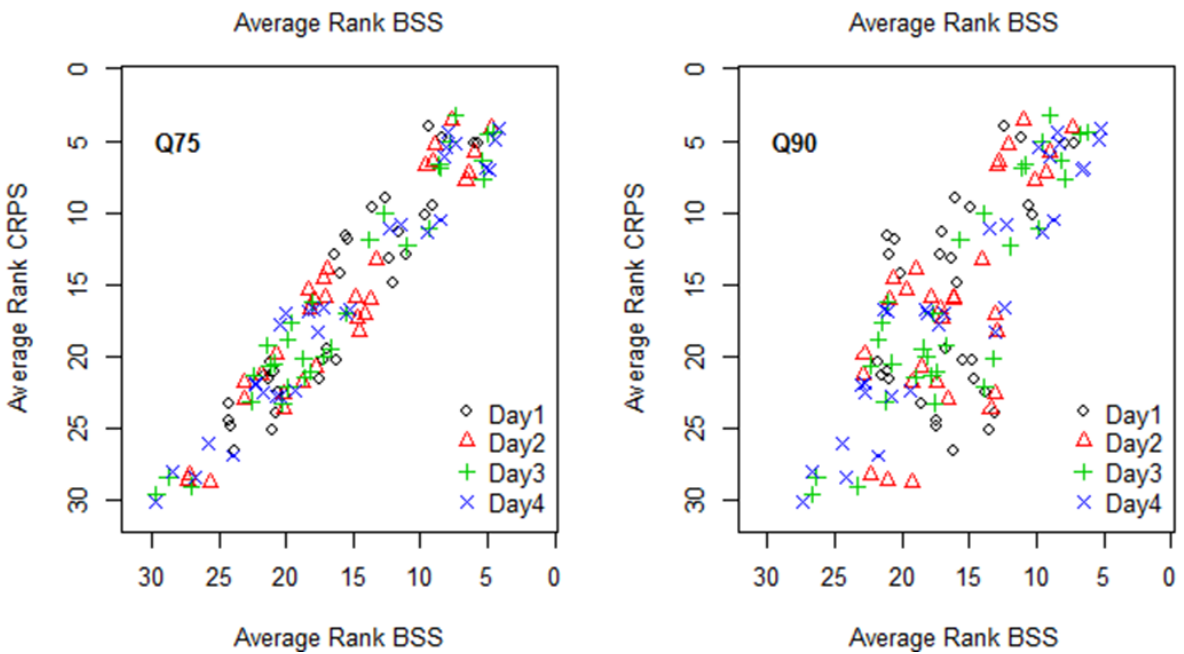

Figure 9. Comparing average rank across 82 gauges based on Brier skill score and CRPS. 

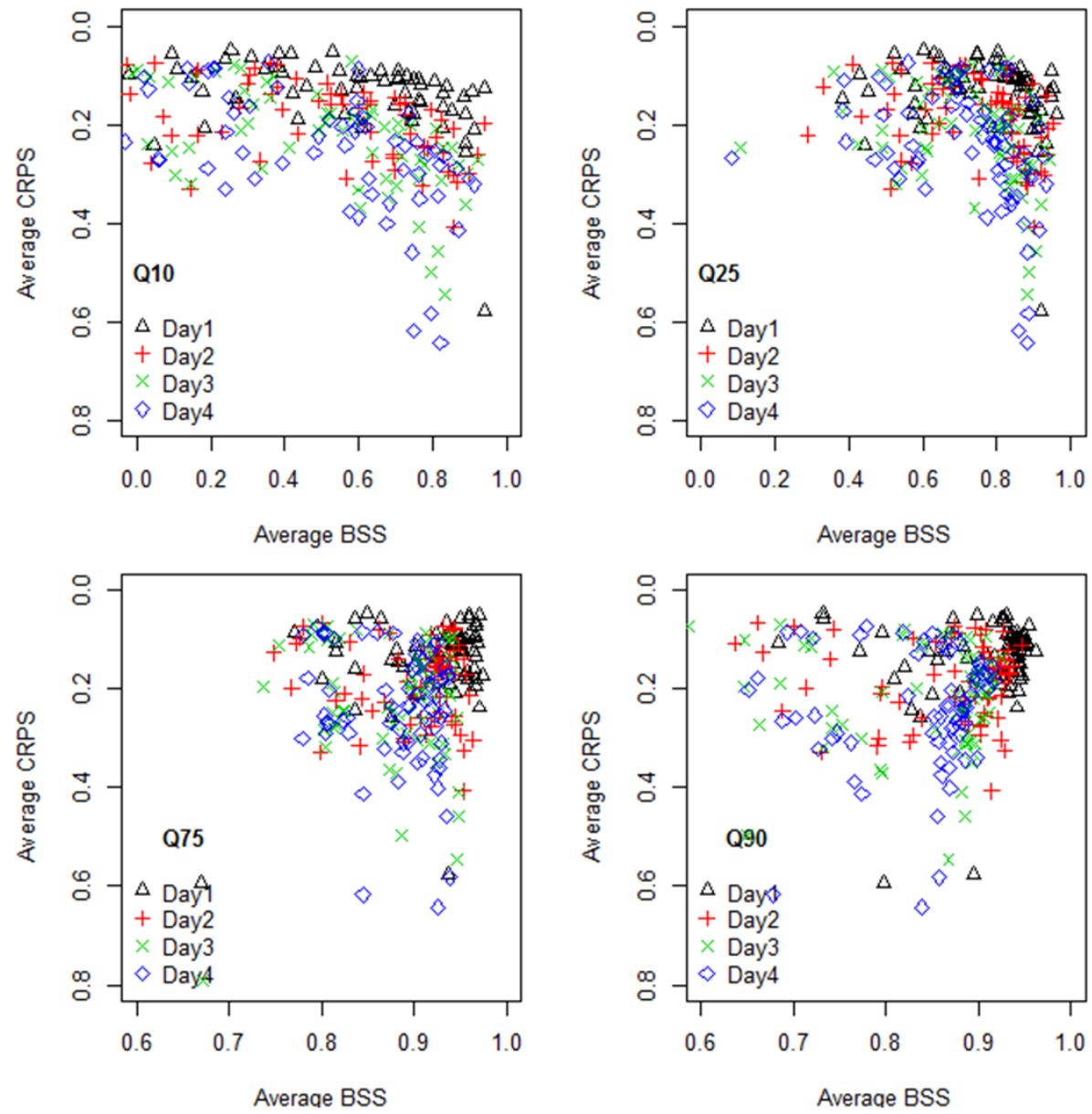

Figure 10. Comparing the performance of combination 30 (err24, err48, rr24, rr48) as measured by Brier skill score and as measured by the continuous ranked probability score. Each data point corresponds with a gauge at a certain lead time. Since the CRPS' perfect score equals zero, the $y$ axis has been reversed.

butions are different. Unlike the dependent variable (forecast error), the forecasts are highly skewed towards the left, because low water levels occur more frequently. Due to its skewed distribution, the forecast becomes a better predictor in a quantile regression predicting a normally distributed dependent variable after a NQT transformation, as successfully used by Weerts et al. (2011). Without a transformation into the normal domain, the scatterplot of forecast and forecast error does not show obvious quantile trends (Fig. 11a). After NQT, the percentiles show distinct quantile trends laid out like a fan (Fig. 12a).

In contrast, errors and rise rates are already approximately normally distributed. No quantile trends can be visually detected after the other four predictors have been subjected to NQT (Fig. 11b-e). In sum, forecast performance in this study is better without NQT, because four of the five independent variables were approximately normally distributed already. Further research is necessary to reconcile predictors with different distributions. Possible solutions could be to define QR configurations for subsets of the transformed dependent and independent variables or to experiment with subjecting only some but not all predictors to NQT.

\subsection{Improvement in forecast performance}

Using the best-performing joint predictor at each river gauge gives an upper bound of the BSSs that can be achieved at best. Confirming the Wood et al. (2009) findings, additionally including the rates of rise and forecasts errors as independent variables into the QR configuration improves the BSS significantly. Figure 13 illustrates the BSS when using the forecast as the only predictor, as studied by Weerts et al. (2011), while Fig. 14 shows the performance for the best joint predictor at each gauge.

Figures 13-15 indicate that the QR method performs better for higher than for lower water levels. Due to the skewed distribution of water levels, the ranges between percentiles in the left tail (lower water levels) correspond with much smaller ranges of water levels (in feet) than in the right tail. Therefore, achieving a good performance in forecasting ex- 

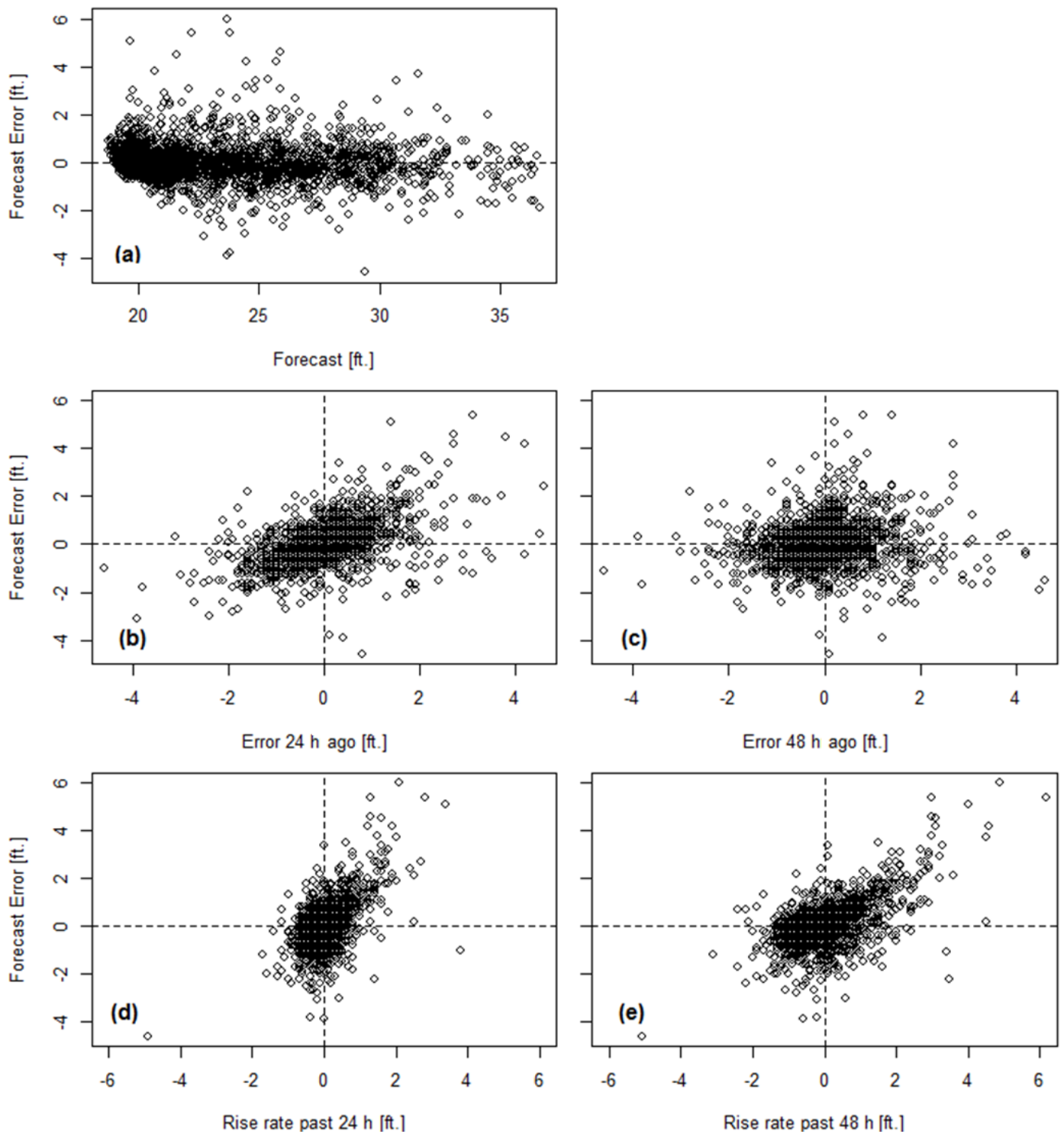

Figure 11. Independent variables plotted against the forecast error for Hardin, IL, with 3 days of lead time. First row: forecast; second row: past forecast errors; third row: rates of rise.

ceedance probabilities of low event thresholds requires much better prediction of the forecast error in feet than for higher event thresholds.

Additionally, Figs. 13-15 show that forecast performance also decreases with increasing lead time, because variables such as rates of rise and past forecast error become proportionally less representative with lead time.

Paired $t$ tests for each combination of lead time and event threshold indicate that using the best joint predictor at each gauge increased average BSS across all gauges statistically significantly (Table 3 ). The performance improves most where forecasts tend to perform worse. The average increase in BSS is largest for extreme water levels, most notably for moderate and major flood stages and for the 10th percentile of water levels (Table 3). The average increase of BSS for a major flood stage is even larger than one, meaning that frequently the method did not have skill before, i.e., negative BSSs. Additionally, predictions with longer lead times experience larger increases in BSS. Compared to using only the forecast as an independent variable, using the best combinations of forecast, rates of rise and past forecast errors as predictors at each gauge not only increases the mean BSS but also decreases the standard deviation of skill scores across gauges, i.e., performance becomes more consistent (Figs. 13, 14).

As expected, the CRPS improves as well when using the best joint predictor at each gauge instead of the forecast as the only predictor. The average CRPS and its standard 

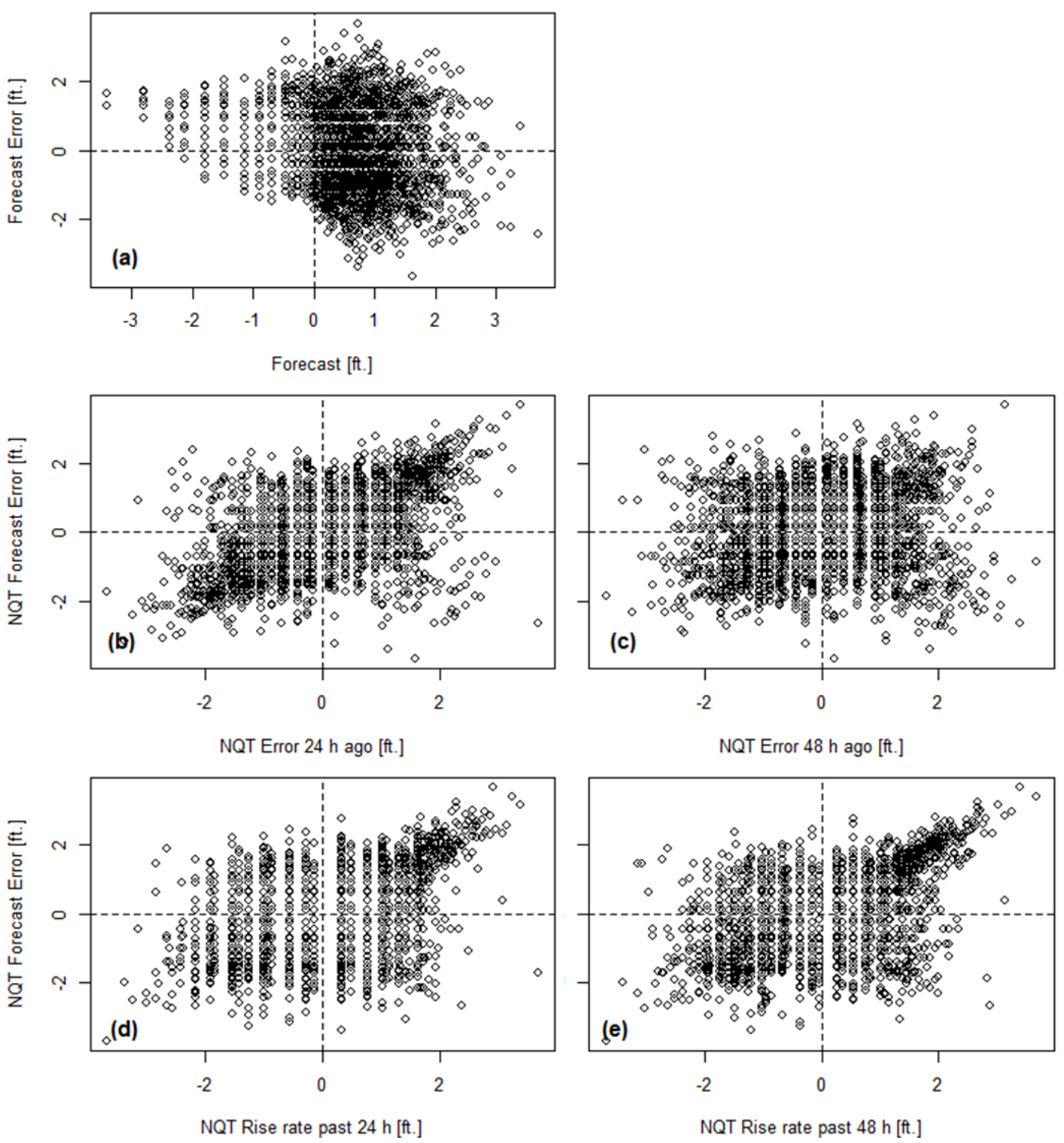

Figure 12. Independent variables after transforming into the Gaussian domain plotted against the forecast error for Hardin, IL, with 3 days of lead time. First row: forecast; second row: past forecast errors; third row: rates of rise.

deviation decrease. The improvement is more pronounced for longer lead times (Fig. 16). Moving away from average CRPS, Table 4 reveals that the best joint predictors for high event thresholds $\left(Q_{75}, Q_{90}\right)$ do not benefit the average CRPS. The fact that the average CRPS does not improve implies that the best joint predictors for high event thresholds increase forecast performance less for high event thresholds than it worsens performance for low event thresholds. The best joint predictors for low event thresholds $\left(Q_{10}, Q_{25}\right)$ do improve average CRPS, so they must be improving the forecast so substantially that the average CRPS increases, even though those best predictors might not perform well for high event thresholds. This is congruent with the finding that average BSS increases much more for percentiles $Q_{10}$ and $Q_{25}$ than for $Q_{75}$ and $Q_{90}$, as shown in Table 3. This reinforces the finding that separate QR models should be configured for individual event thresholds based on the BSS, rather than for the whole distribution based on the CRPS.

The fact that the Brier score can be decomposed into reliability, resolution and uncertainty allows for a closer look at which improvements are being achieved by including more predictors than just the forecast. Table 4 summarizes the results of paired $t$ tests comparing the forecast-only and the best-performing joint predictor for each gauge for the components of the BSS as well as the CRPS.

The Brier score and the Brier skill score mainly improve, because the resolution increases when using the bestperforming set of independent variables at each gauge (Ta- 
Table 3. Results of paired $t$ tests comparing the QR method's performance with only forecast as predictor and the best-performing combination of five predictors for each river gauge.

\begin{tabular}{|c|c|c|c|c|c|c|c|c|c|c|c|c|c|c|c|c|}
\hline & \multicolumn{4}{|c|}{1 day } & \multicolumn{4}{|c|}{2 days } & \multicolumn{4}{|c|}{3 days } & \multicolumn{4}{|c|}{4 days } \\
\hline & Diff. & $T$ stat. & $\mathrm{Df}^{*}$ & $p$ val. & Diff. & $T$ stat. & $\mathrm{Df}^{*}$ & $p$ val. & Diff. & $T$ stat. & $\mathrm{Df}^{*}$ & $p$ val. & Diff. & $T$ stat. & $\mathrm{Df}^{*}$ & $p$ val. \\
\hline$Q_{10}$ & 0.20 & 8.68 & 80 & .000 & 0.25 & 8.98 & 79 & .000 & 0.28 & 8.53 & 79 & .000 & 0.27 & 10.08 & 79 & .000 \\
\hline$Q_{25}$ & 0.13 & 6.06 & 81 & .000 & 0.15 & 7.10 & 81 & .000 & 0.18 & 9.00 & 80 & .000 & 0.20 & 11.35 & 80 & .000 \\
\hline$Q_{75}$ & 0.03 & 10.19 & 81 & .000 & 0.05 & 9.58 & 81 & .000 & 0.08 & 11.00 & 81 & .000 & 0.12 & 10.80 & 81 & .000 \\
\hline$Q_{90}$ & 0.03 & 8.38 & 81 & .000 & 0.06 & 9.33 & 81 & .000 & 0.10 & 10.54 & 81 & .000 & 0.15 & 11.95 & 81 & .000 \\
\hline Action & 0.05 & 7.76 & 72 & .000 & 0.14 & 2.37 & 73 & .010 & 0.14 & 5.39 & 73 & .000 & 0.18 & 7.30 & 73 & .000 \\
\hline Minor & 0.40 & 2.98 & 60 & .002 & 0.35 & 3.37 & 60 & .001 & 0.37 & 3.70 & 60 & .000 & 0.51 & 4.35 & 62 & .000 \\
\hline Moderate & 0.44 & 2.93 & 41 & .003 & 0.52 & 2.94 & 42 & .003 & 0.81 & 3.97 & 45 & .000 & 0.74 & 5.08 & 47 & .000 \\
\hline Major & 1.36 & 3.00 & 19 & .004 & 1.84 & 4.27 & 22 & .000 & 2.14 & 4.85 & 26 & .000 & 1.80 & 6.01 & 34 & .000 \\
\hline
\end{tabular}

* Df means degrees of freedom.

Brier Skill Score

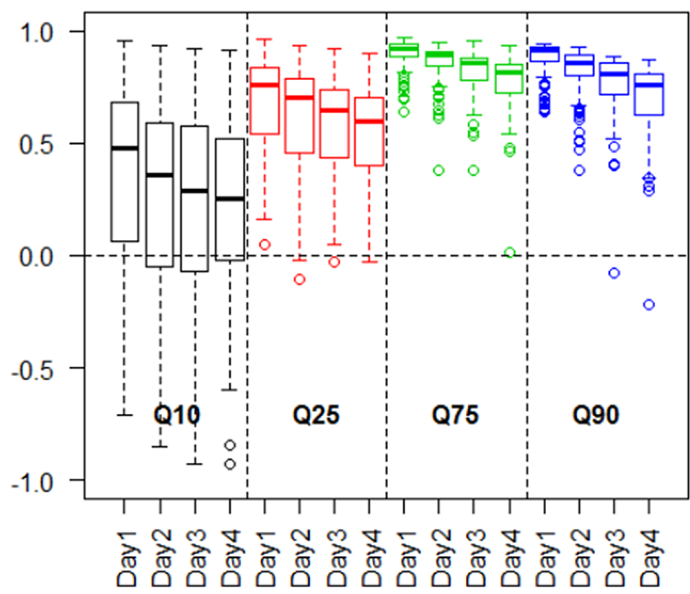

Figure 13. BSS for the forecast-only configuration for different lead times and event thresholds. The BSS' perfect score equals one. A BSS of zero indicates a forecast without skill.

ble 4). Visualizing the improvement in forecast performance for a lead time of 3 days and the 75th percentile threshold $\left(Q_{75}\right)$, Fig. 17 illustrates that the forecast-only QR configuration as studied by Weerts et al. (2011) has high reliability (i.e., the reliability is close to zero). So reliability improves statistically significantly for lower water levels $\left(Q_{10}, Q_{25}\right)$, but the magnitude of improvement in reliability is 1 order of magnitude smaller than the improvement in resolution (Table 4).

\subsection{One-size-fits-all approach - Brier skill score}

Combing these findings, the configurations for the various river gauges can generally be based on the same joint predictor of the four independent variables excluding the forecast itself (combination 30). But for extremely high water levels, a configuration specific to each river gauge has to be built in order to achieve high BSSs.

\section{Brier Skill Score}

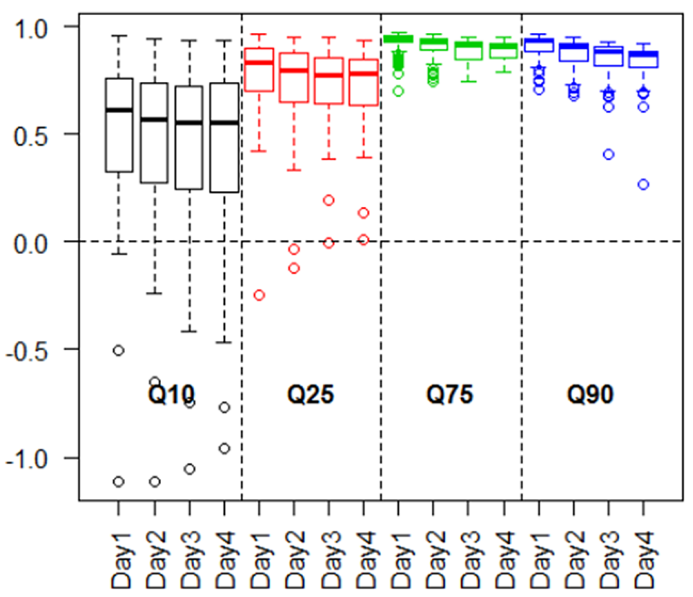

Figure 14. BSS for the best-performing joint predictor at each gauge for different lead times and event thresholds. The BSS' perfect score equals one. A BSS of zero indicates a forecast without skill.

Verifying this finding, a one-size-fits-all approach was tested to investigate whether customizing the QR configuration to each river gauge would be worth it. The rates of rise in the past 24 and $48 \mathrm{~h}$ and the forecast errors 24 and $48 \mathrm{~h}$ ago (combination 30 in Table 1) serve as independent variables for this approach. This combination of predictors has been chosen, because it performed well for most gauges (see Sect. 4.1). Furthermore, less important predictors in the combination will get small coefficients in the quantile regression. So additional variables are unlikely to do harm but can improve the estimates at various stages. The price of opting for a joint predictor with more variables is an increase of the risk of overfitting.

Paired $t$ tests have been executed to investigate whether this one-size-fits all approach performs statistically significantly worse than using the best combination of predictors for each gauge. It was found that this approach on av- 
Table 4. Results of paired $t$ tests comparing the QR method's performance with only forecast as predictor and the best-performing combination of five predictors for each river gauge for the Brier score.

\begin{tabular}{llccccl}
\hline $\begin{array}{l}\text { Event } \\
\text { threshold }\end{array}$ & $\begin{array}{l}\text { Lead } \\
\text { time }\end{array}$ & $\begin{array}{c}\text { Brier } \\
\text { score }\end{array}$ & $\begin{array}{c}\text { Brier } \\
\text { skill score }\end{array}$ & Reliability & Resolution & CRPS \\
\hline \multirow{4}{*}{$Q_{10}$} & 1 day & $-.012^{* * *}$ & $.20^{* * *}$ & $-.002^{* * *}$ & $.008^{* * *}$ & $-.026^{* * *}$ \\
& 2 days & $-.014^{* * *}$ & $.25^{* * *}$ & $-.002^{* * *}$ & $.010^{* * *}$ & $-.082^{* *}$ \\
& 3 days & $-.016^{* * *}$ & $.28^{* * *}$ & $-.002^{* * *}$ & $.012^{* * *}$ & $-.121^{* * *}$ \\
& 4 days & $-0.17^{* * *}$ & $.27^{* * *}$ & $-.001^{*}$ & $.013^{* * *}$ & -.054 \\
\hline \multirow{6}{*}{$Q_{25}$} & 1 day & $-.018^{* * *}$ & $.13^{* * *}$ & $-.003^{* * *}$ & $.013^{* * *}$ & $-.028^{* *}$ \\
& 2 days & $-.023^{* * *}$ & $.16^{* * *}$ & $-.002^{* * *}$ & $.018^{* * *}$ & $-.088^{* *}$ \\
& 3 days & $-.027^{* * *}$ & $.18^{* * *}$ & $-.003^{* * *}$ & $.021^{* * *}$ & $-.097^{* *}$ \\
$Q_{75}$ & 4 days & $-.031^{* * *}$ & $.20^{* * *}$ & $-.002^{* * *}$ & $.025^{* * *}$ & -.475. \\
& 1 day & $-.005^{* * *}$ & $.03^{* * *}$ & .000 & $.011^{* * *}$ & .342 \\
& 2 days & $-.011^{* * *}$ & $.05^{* * *}$ & -.000. & $.015^{* * *}$ & .009 \\
& 3 days & $-.016^{* * *}$ & $.08^{* * *}$ & -.000 & $.021^{* * *}$ & .188 \\
\hline \multirow{2}{*}{$Q_{90}$} & 4 days & $-.025^{* * *}$ & $.12^{* * *}$ & -.000 & $.028^{* * *}$ & -.064 \\
& 1 day & $-.003^{* * *}$ & $.03^{* * *}$ & $-.000^{* *}$ & $.013^{* * *}$ & .159 \\
& 2 days & $-.005^{* * *}$ & $.06^{* * *}$ & $-.000^{*}$ & $.015^{* * *}$ & $-.086^{* *}$ \\
& 3 days & $-.010^{* * *}$ & $.10^{* * *}$ & -.000 & $.019^{* * *}$ & .163 \\
& 4 days & $-.015^{* * *}$ & $.15^{* * *}$ & $-.000^{*}$ & $.025^{* * *}$ & -.075 \\
\hline
\end{tabular}

$p$ values: $* * *:<0.001 ; * *: 0.01 ; *: 0.05 ; .: 0.1$.
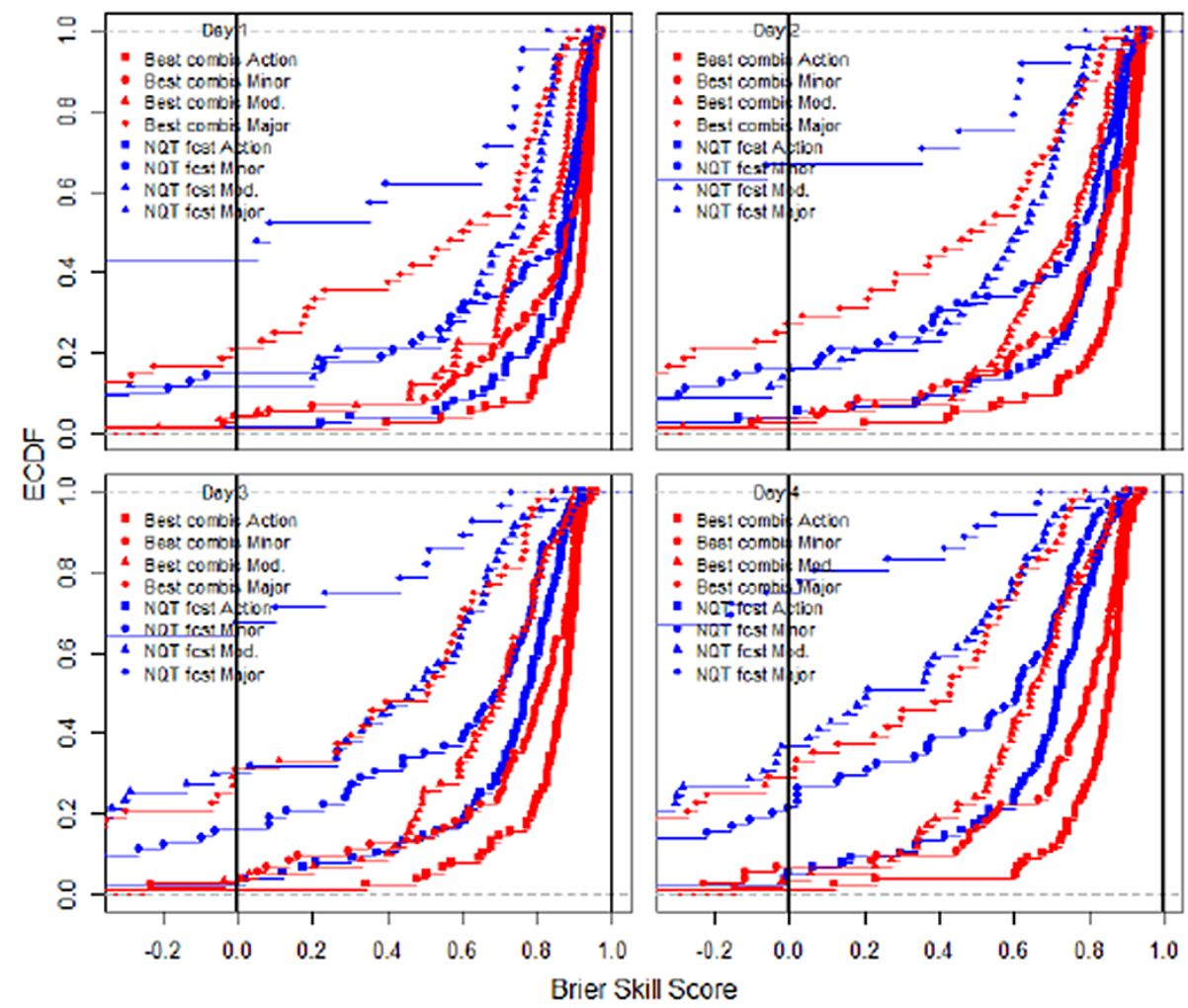

Figure 15. Empirical cumulative density functions of three QR configurations predicting exceedance probabilities of the action, minor, moderate, and major flood stages: the configuration using the transformed forecast as the only independent variable (NQT fcst), and the best-performing combination for each river gauge (upper performance limit; best combis). 
Table 5. Results of paired $t$ test comparing best combinations of predictors with the one-size-fits-all approach.

\begin{tabular}{|c|c|c|c|c|c|c|c|c|c|c|c|c|c|c|c|c|}
\hline & \multicolumn{4}{|c|}{1 day } & \multicolumn{4}{|c|}{2 day } & \multicolumn{4}{|c|}{3 days } & \multicolumn{4}{|c|}{4 days } \\
\hline & Diff. & $T$ stat. & Df & $p$ val. & Diff. & $T$ stat. & Df & $p$ val. & Diff. & $T$ stat. & Df & $p$ val. & Diff. & $T$ stat. & Df & $p$ val \\
\hline$Q_{10}$ & .054 & 4.61 & 79 & .000 & .071 & 5.56 & 79 & .000 & .075 & 6.36 & 79 & .000 & .071 & 7.54 & 79 & .000 \\
\hline$Q_{75}$ & .003 & 6.56 & 81 & .000 & .004 & 7.25 & 81 & .000 & .005 & 4.63 & 81 & .000 & .004 & 6.42 & 81 & .000 \\
\hline$Q_{90}$ & .008 & 7.10 & 81 & .000 & .015 & 4.37 & 81 & .000 & .012 & 5.16 & 81 & .000 & .021 & 1.84 & 81 & .035 \\
\hline Action & .024 & 1.94 & 72 & .028 & .031 & 1.97 & 73 & .026 & .039 & 1.96 & 73 & .027 & .022 & 2.20 & 73 & .016 \\
\hline Major & .245 & 2.09 & 19 & .025 & .212 & 2.34 & 22 & .014 & .234 & 2.66 & 26 & .007 & .375 & 3.25 & 34 & .001 \\
\hline
\end{tabular}

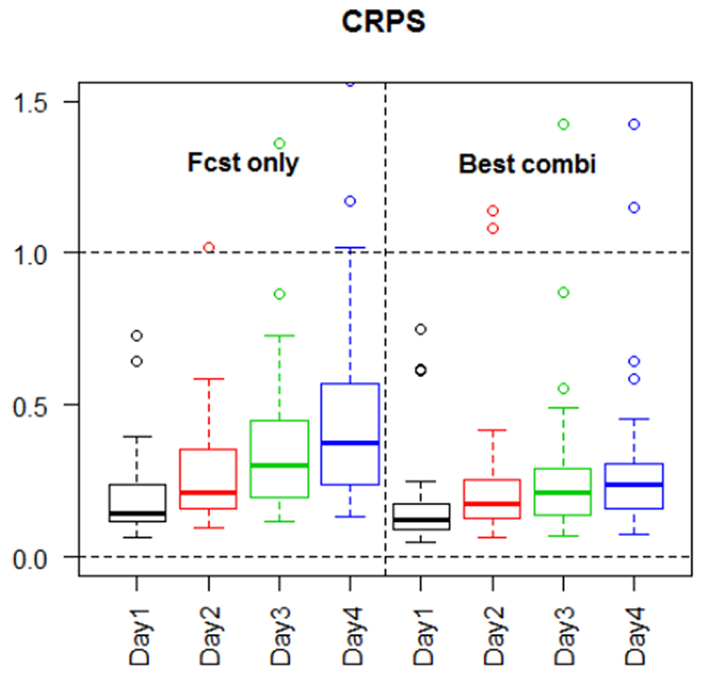

Figure 16. CRPS for the forecast-only configuration and for the best-performing joint predictor at each gauge for different lead times and event thresholds. The CRPS' perfect score equals zero.

erage performs statistically significantly not as well as using the best-performing combination of predictors. But the difference in average BSS is small, ranging between 0.003 and 0.075 (Table 5).

However, using the best joint predictors results in a much better performance for major flood stages than the one-sizefits-all approach. The average difference between average BSSs amounts to $0.21-0.38$ (Table 5). Given that a BSS for a forecast with skill ranges between one and zero, this is a substantial difference. In sum, the same joint predictor can be used for all river gauges without much loss in performance, except for extremely high water levels.

\subsection{Robustness}

\subsubsection{Minimum length of training data set}

Stationarity cannot always be assumed (Milly et al., 2008). River regimes can change through natural processes like sed-

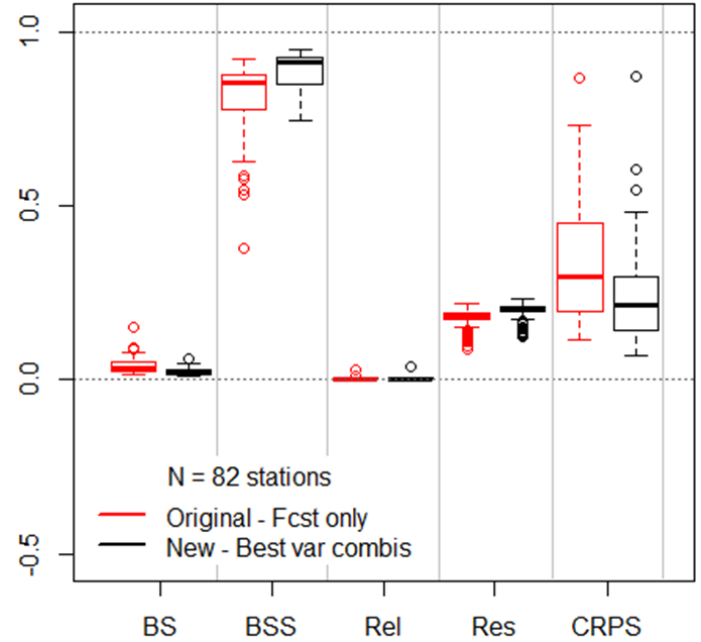

Figure 17. Comparison of the forecast-only $Q R$ configuration (i.e., only transformed forecast as independent variables) and using the best-performing joint predictor at each gauge along various measures of forecast quality: BS, BSS, reliability (Rel), resolution (Res), and CRPS. Lead time of 3 days and 75th percentile of observation levels as threshold.

imentation or human intervention. Those changes can occur gradually or as step changes. This analysis of robustness is meant to determine the minimum length of the training data set to be able to produce skillful forecasts again after a step change using the QR method. Additionally, the analysis is meant to find out to which length the forecaster should limit the training data set when gradual change is occurring. After all, in such a case, each year further in the past is less representative of the year ahead, so the training data set should be as short as possible.

The impact of the length of the training data set on the configuration's performance measured by the BSS was assessed for the best joint predictor (i.e., rates of rise and forecast errors as independent variables for all gauges) for Hardin and Henry on the Illinois River. Each year between 2003 and 2013 was forecast by QR configurations trained on however many years of archived forecasts were available in that year, 

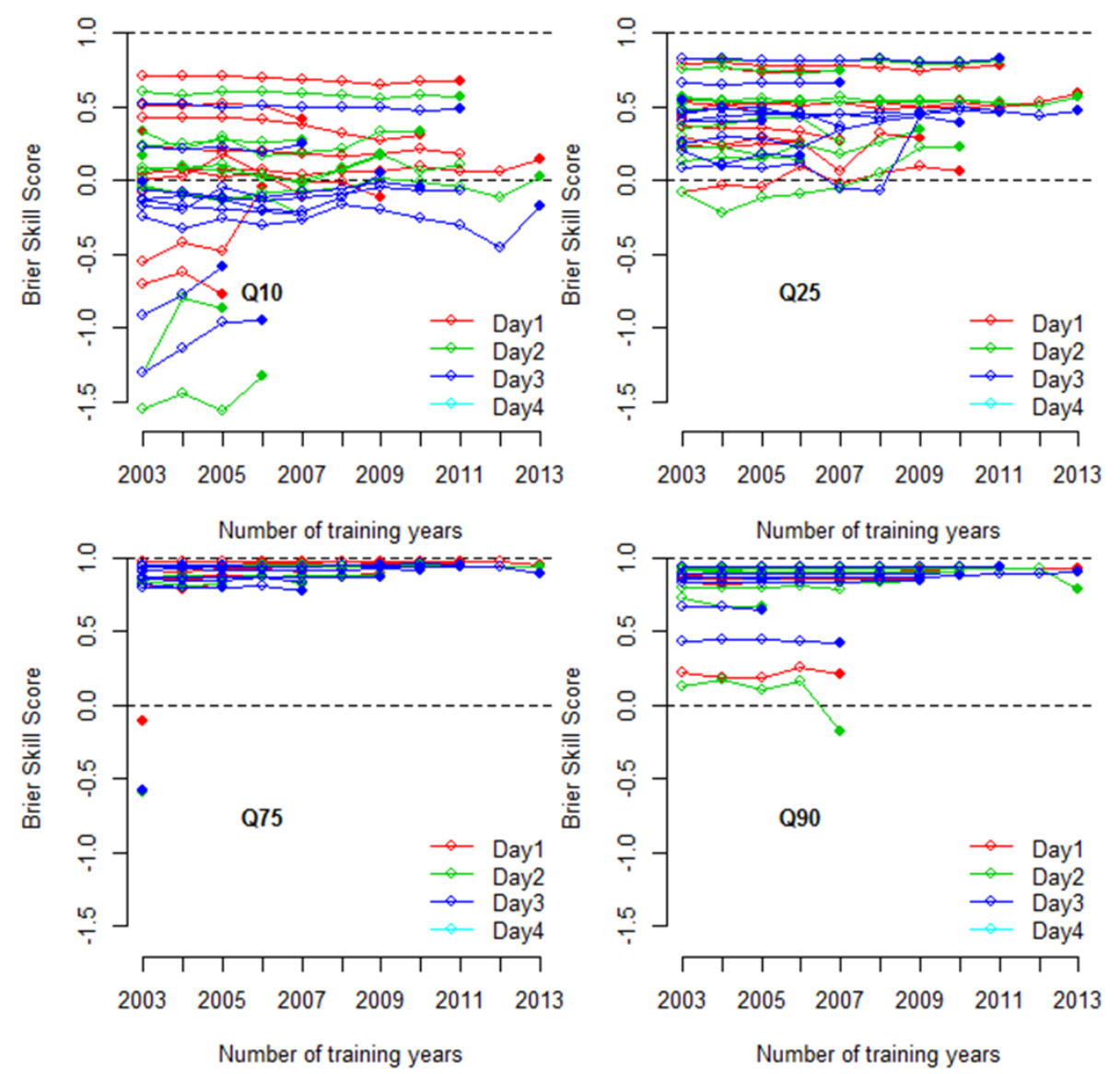

Figure 18. Brier skill score for various forecast years and various sizes of the training data set across different lead times (colors) and event thresholds (plots) for Hardin, IL (HARI2). The filled-in end point of each line indicates the BSS for the forecast year on the $x$ axis with 1 year in the training data set. Each point to the left stands for 1 additional training year for that same forecast year.

i.e., the forecasts for 2005 are produced by a model trained on less data than those for 2013. Then, the BSS for that year (e.g., 2005 or 2013) was computed.

Figures 18 and 19 show that at Henry and Hardin it barely matters for the BSS how many years are included in the training data set. This finding is congruent with the fact that Weerts et al. (2011) were able to achieve outstanding results with the QR method using training data sets that were only 2 years long. Only needing short time series to define a skillful QR configuration implies (i) skillful forecasts can be produced not long after a step change and that (ii) the configuration parameters can be updated regularly so that gradually changing relationships between predictors, for example, can be taken into account.

\subsubsection{Sensitivity analysis}

Furthermore, we aim at identifying the factors that impact forecast skill as quantified by the BSS and at generalizing the result regarding training data length described for Hardin and Henry above. To do so, the same analysis as for Hardin and Henry was repeated for all 82 gauges. Following that, a regression analysis was executed with the BSS as the dependent variable and event thresholds $\left(Q_{10}, Q_{25}, Q_{75}, Q_{90}\right)$, the river gauges and forecast years as independent nominal variables, and the lead time (1-4 days) and number of training years as independent ratio variables. This regression is meant to identify the factors to which the forecast performance as measured by the BSS is sensitive to, i.e., which factors statistically significantly impact forecast performance.

The forecast performance was found to vary statistically significantly across all tested dimensions, except the number of training years (Table 6). This results in a very wide range of BSSs (Figs. 13, 14). Accordingly, for the user, it is particularly difficult to know how much to trust a forecast if the performance depends so much on context. Likewise, this is the case for the QR configuration based on the forecast only (not shown).

A closer look at the regression coefficients (Table 6) provides interesting insights. For low event thresholds, the BSSs are much worse than for high thresholds. As mentioned above, for such low event thresholds the forecast has to pre- 

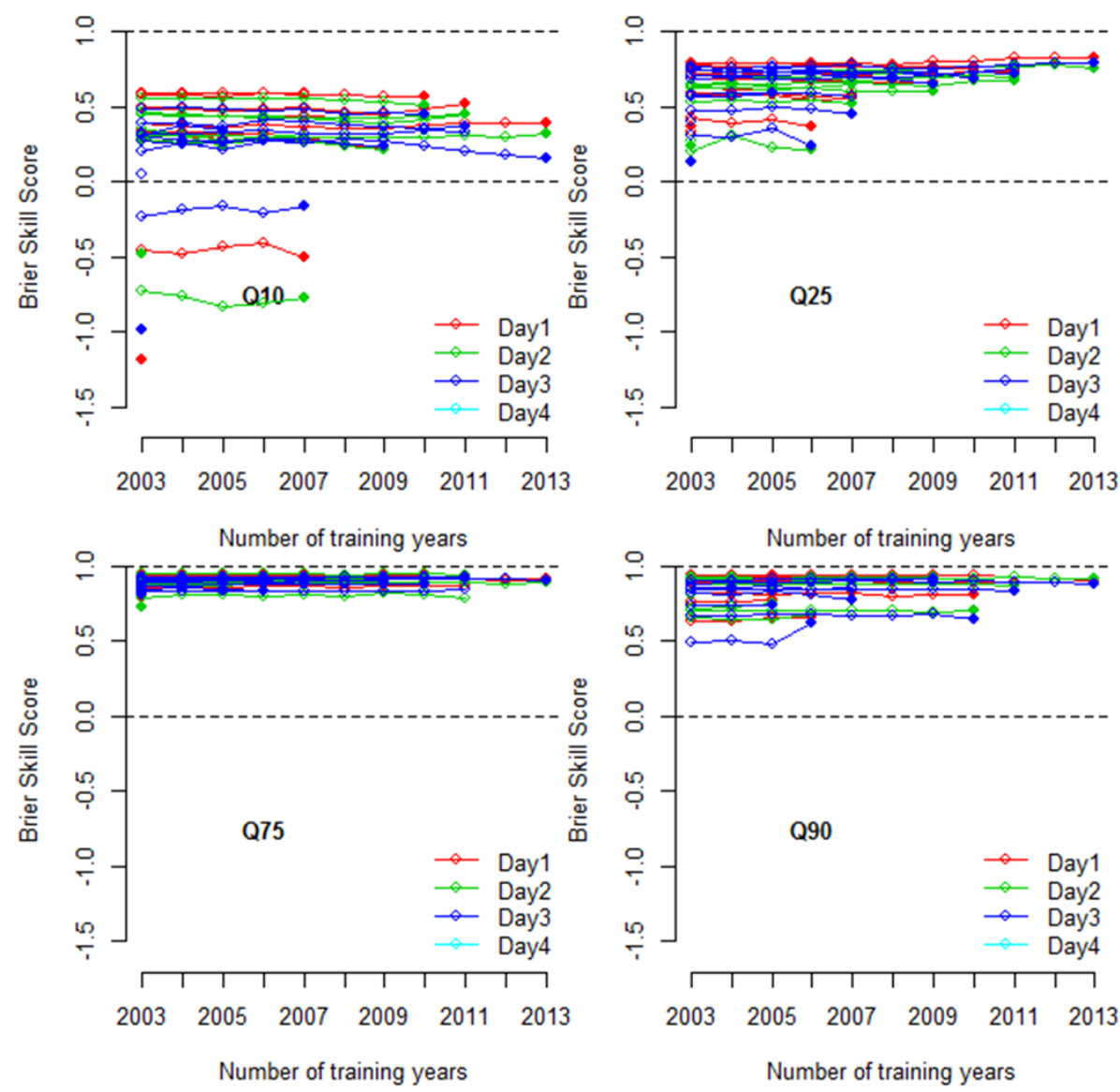

Figure 19. Brier skill score for various forecast years and various sizes of the training data set across different lead times (colors) and event thresholds (plots) for Henry, IL (HNYI2). The filled-in end point of each line indicates the BSS for the forecast year on the $x$ axis with 1 year in the training data set. Each point to the left stands for 1 additional training year for that same forecast year.

dict the water levels much more accurately to achieve a similar forecast performance than for higher water levels, due to the skewed distribution of water levels. In the lower tail, each percentile corresponds with a much shorter span of water levels than in the upper tail. Using a higher resolution in the lower tail is therefore advisable.

As expected, the BSSs slightly decrease with lead time, because independent variables such as rates of rise and past forecast error gradually become less representative of the days to be forecasted.

Regarding the forecast quality for each forecast year, the regression is slightly biased. The earlier years are included less often in the data set, having on average less years of data in their training data set because, for example, unlike for the year 2013, 10 years of training data were not available for the year 2006. Nonetheless, the regression indicates that 2008 was particularly difficult to forecast and 2012 relatively easy, i.e., they are associated with relatively low and high coefficients respectively (Table 6).

The performance of the forecast additionally depends on the river gauge. The coefficients of the river gauges, included as factors in the regression, have been excluded from Table 6 for the sake of brevity. Instead, Fig. 20 maps the geographic position of the river gauges with the color code indicating each gauge's regression coefficient. The coefficient indicates the method's performance at the particular gauge as compared to the average performance. The coefficients are lower and therefore the Brier skill scores are lower for gauges far upstream a river, off the main stream, and those close to confluences.

Precipitation is one of the major sources of uncertainty in river forecasting. For example, if rainfall shifts by a few miles it might be raining down in a different river basin. This makes rises in water level difficult to anticipate, making rates of rise such a successful predictor of the distribution of forecast errors. However, upstream and close to confluences, rates of rise and past forecast errors perform less well as predictors than elsewhere. This suggests that uncertain expected rainfall constitutes a smaller part of the overall uncertainty.

Close to confluences, the joining second river adds a major part of that additional uncertainty. The interaction between the rivers increases uncertainty, in addition to the uncertainty 


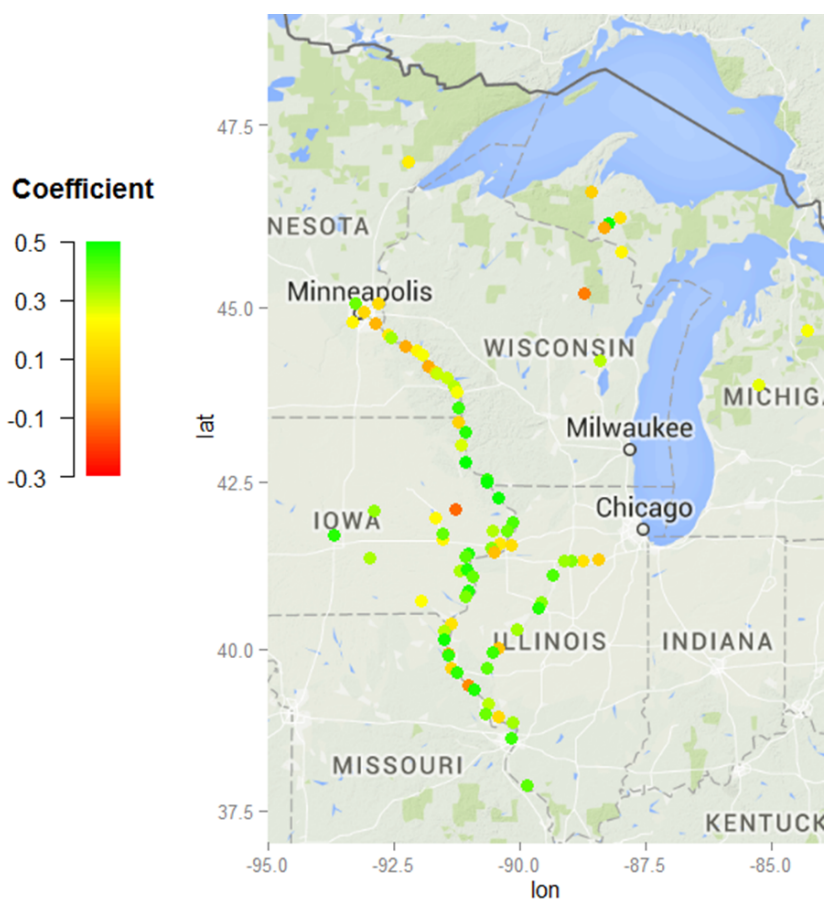

Figure 20. Geographical position of rivers. Colors indicate the regression coefficient of each station with the Brier skill score as dependent variable.

associated with the joining river itself, e.g., the uncertain expected rainfall along its course. At upstream gauges, the rates of rise possibly provide less information, because due to smaller basin sizes concentration times are shorter, i.e., water levels rise quicker. In this case, the rise in water level of the past 24 and $48 \mathrm{~h}$ may not sufficiently capture rises occurring with shorter notice. The argument holds for forecast errors as well. If concentration times are short, the forecast error of $48 \mathrm{~h}$ ago is not representative of those in the near future.

\section{Conclusions}

In this study, QR has been applied to estimate the probability of the river water level exceeding various event thresholds (i.e., 10th, 25th, 75th, and 90th percentiles of observed water levels as well as the four flood stages of each river gauge). It further develops the application of $\mathrm{QR}$ for estimating river forecast uncertainty (a) comparing different sets of independent variables and (b) testing the technique's robustness across locations, lead times, event thresholds, forecast years and sizes of the training data set.

When compared to the configuration using only the forecast, it was found that including rates of rise in the past 24 and $48 \mathrm{~h}$ and the forecast errors of 24 and $48 \mathrm{~h}$ ago as independent variables improves the performance of the $\mathrm{QR}$ configuration, as measured by the Brier skill score. This con-
Table 6. Regression results of sensitivity analysis.

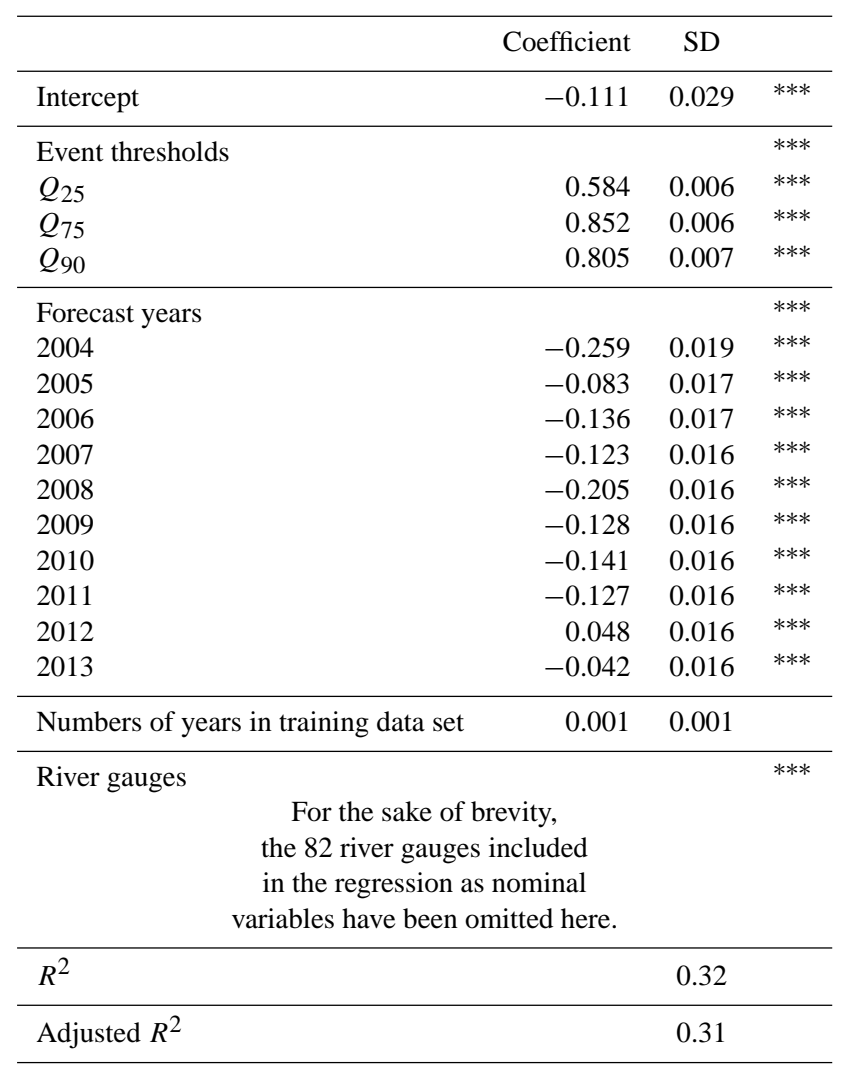

$p$ values: ${ }^{* * *}:<0.001 ;^{* *}: 0.01 ;^{*}: 0.05 ;():$.

firms Wood et al.'s (2009) finding that rate of rise is a valuable predictor for $\mathrm{QR}$ error models. The configuration with the forecast as the only independent variable, as studied by Weerts et al. (2011), produced estimates with high reliability. Including the other four predictors mentioned above mainly increases the resolution.

For extremely high water levels, the combinations of independent variables that perform best vary across stations. On those days, combinations of fewer independent variables perform better than those that include more. The most likely explanation is that QR configurations based on large joint predictors result in overfitting the data. In contrast to these extremely high event thresholds, larger sets of predictors work better than smaller ones for non-extreme and low event thresholds. Additionally, customizing the set of predictors to the event thresholds does not improve the BSS much, except for extremely high event thresholds, i.e., major flood stage.

When forming a joint predictor, the independent variables, rates of rise and forecast errors, do not combine well with the forecast itself, because the forecast has a skewed distribution while the other predictors are approximately normally distributed. The forecast becomes an excellent predictor for linear quantile regression after NQT. However, the other four variables lose their value as predictors when subjected to 
NQT, because their original distribution is already approximately normal. Therefore, it is difficult to combine predictors with different distributions. A possible solution could be to define QR configurations for subsets of the transformed data or to experiment with only subjecting some of the predictors to NQT.

This study shows the importance of configuring QR models for individual event thresholds rather than using one configuration to estimate the whole forecast distribution. The tails are too different to use the same joint predictors and parametrization.

The studied QR configurations are relatively robust to the size of training data set, which is convenient if stationarity cannot be assumed (Milly et al., 2008), a step change in the river regime has occurred or - as is the case for most river forecast centers - only recent forecast data have been archived. However, the performance of the technique depends heavily on the river gauge, the lead time, event threshold and year that are being forecast. This results in a very wide range of Brier skill scores. This means that the danger remains that forecast users make good experiences with a forecast one year or at one location and assume it is equally reliable in other locations and every year. As is the case with most other forecasts, an indication of forecast uncertainty needs to be communicated alongside the exceedance probabilities generated by our approach.

As is the case for many forecasting methods, the studied QR configurations perform less well for longer lead times, extreme event thresholds that are characterized by data scarcity, and for gauges far upstream a river, off the main stream or close to confluences where different factors interact with each other. Additionally, QR configurations underperform for low event thresholds. Due to the skewed distribution of water levels, forecasts have to perform better in estimating low water levels to achieve the same BSSs as for high event thresholds, because in the lower tail each percentile spans a smaller range of water levels. Using a higher resolution in the lower tail would probably improve the forecast performance for low event thresholds.

\section{Future work}

This technique can be further developed in several ways to achieve higher Brier skill scores and more robustness. First, more independent variables can be added. Observed precipitation, the precipitation forecast (i.e., POP - probability of precipitation) and the upstream water levels are promising candidates, because the forecast used in this study includes the precipitation forecast for only the next $12 \mathrm{~h}$. However, currently, the precipitation data and forecasts can only be requested in chunks of a month, three chunks per day, from the NCDC's HDSS Access System. For a period of 12 years, requesting such data for several weather stations is obviously time consuming, not least because the geographical units of the weather forecasts bulletins do not correspond with those of the river forecast bulletins. Upstream water levels can easily be included after manually determining the upstream gauge(s) for each of the 82 NCRFC gauges. To improve performance at gauges close to river confluences, off the main stream and upstream water levels of the gauges on the joining river should be included as well.

However, note that many hydrological variables have a skewed distribution, so that they cannot readily be combined into a joint predictor with normally distributed variables such as rates of rise and past forecast errors, as used in this study. Future work should focus on reconciling predictors with different distributions.

Different approaches of sub-setting the data to improve performance also warrant consideration in boosting the performance of the QR method. Particularly, clustering the data by variability seems promising.

Additionally, the studied technique would need to be verified for gauges for which the NCRFC does not publish daily forecasts. Ignorance of the uncertainty inherent in river forecasts has had some of the most unfortunate impacts on decision-making in Grand Forks, ND, and Fargo, ND (Pielke, 1999; Morss, 2010). Both of those stages are discontinuously forecasted NCRFC gauges.

Finally, this paper uses a brute force approach by simply calculating and comparing all possible combinations of independent variables. A mathematically more challenging stepwise quantile regression would not only be more elegant but would also provide better safeguards against overfitting the data.

Acknowledgements. Many thanks to Grant Weller, who suggested looking into quantile regression to predict forecast errors. We would like to thank the three reviewers for their insightful comments. The paper greatly benefitted from their comments. As to funding, Frauke Hoss is supported by an ERP fellowship of the German National Academic Foundation and by the Center of Climate and Energy Decision Making (SES-0949710), through a cooperative agreement between the National Science Foundation and Carnegie Mellon University (CMU).

Edited by: A. Weerts

\section{References}

Alexander, M., Harding, M., and Lamarche, C.: Quantile Regression for Time-Series-Cross-Section-Data, Int. J. Stat. Manage. Syst., 4, 47-72, 2011.

Bogner, K., Pappenberger, F., and Cloke, H. L.: Technical Note: The normal quantile transformation and its application in a flood forecasting system, Hydrol. Earth Syst. Sci., 16, 1085-1094, doi:10.5194/hess-16-1085-2012, 2012.

Brier, G. W.: Verification of Forecasts Expressed in Terms of Probability, Mon. Weather Rev., 78, 1-3, doi:10.1175/15200493(1950)078<0001:VOFEIT>2.0.CO;2, 1950. 
Demargne, J., Wu, L., Regonda, S. K., Brown, J. D., Lee, H., He, M., Seo, D.-J., Hartman, R., Herr, H. D., Fresch, M., Schaake, J., and Zhu, Y.: The Science of NOAA's Operational Hydrologic Ensemble Forecast Service, B. Am. Meteorol. Soc., 95, 79-98, doi:10.1175/BAMS-D-12-00081.1, 2013.

Hsu, W. and Murphy, A. H.: The attributes diagram A geometrical framework for assessing the quality of probability forecasts, Int. J. Forecast., 2, 285-293, doi:10.1016/0169-2070(86)900488, 1986.

Illinois Department of Natural Resources: Aquatic Illinois - Illinois Rivers and Lakes Fact Sheets, available at: http://dnr.state. il.us/education/aquatic/aquaticillinoisrivlakefactshts.pdf (last access: 3 February 2015), 2011.

Jolliffe, I. T. and Stephenson, D. B.: Forecast Verification: A Practitioner's Guide in Atmospheric Science, John Wiley \& Sons, doi:10.1002/9781119960003.fmatter/pdf, 2012.

Kelly, K. S. and Krzysztofowicz, R.: A bivariate meta-Gaussian density for use in hydrology, Stoch. Hydrol. Hydraul., 11, 1731, doi:10.1007/BF02428423, 1997

Koenker, R.: Quantile Regression, Cambridge University Press, 2005.

Koenker, R.: quantreg: Quantile Regression, R Package Version 505, available at: http://CRAN.R-project.org/package= quantreg (last access: 27 August 2014), 2013.

Koenker, R. and Bassett, G.: Regression Quantiles, Econometrica, 46, 30-50, doi:10.2307/1913643, 1978.

Koenker, R. and Machado, J. A. F.: Goodness of Fit and Related Inference Processes for Quantile Regression, J. Am. Stat. Assoc., 94, 1296-1310, doi:10.1080/01621459.1999.10473882, 1999.

Leahy, C. P.: Objective Assessment and Communication of Uncertainty in Flood Warnings, 5th Flood Management Conference, 9-12 October 2007, Warrnamboll, 2007.

López López, P., Verkade, J. S., Weerts, A. H., and Solomatine, D. P.: Alternative configurations of quantile regression for estimating predictive uncertainty in water level forecasts for the upper Severn River: a comparison, Hydrol. Earth Syst. Sci., 18, 34113428, doi:10.5194/hess-18-3411-2014, 2014.

Milly, P. C. D., Betancourt, J., Falkenmark, M., Hirsch, R. M., Kundzewicz, Z. W., Lettenmaier, D. P., and Stouffer, R. J.: Stationarity Is Dead: Whither Water Management?, Science, 319, 573-574, doi:10.1126/science.1151915, 2008.

Montanari, A. and Brath, A.: A stochastic approach for assessing the uncertainty of rainfall-runoff simulations, Water Resour. Res., 40, W01106, doi:10.1029/2003WR002540, 2004.

Montanari, A. and Grossi, G.: Estimating the uncertainty of hydrological forecasts: A statistical approach, Water Resour. Res., 44, W00B08, doi:10.1029/2008WR006897, 2008

Morss, R. E.: Interactions among Flood Predictions, Decisions, and Outcomes: Synthesis of Three Cases, Nat. Hazards Rev., 11, 8396, doi:10.1061/(ASCE)NH.1527-6996.0000011, 2010.

National Climatic Data Center: HDSS Access System, available at: http://cdo.ncdc.noaa.gov/pls/plhas/HAS.FileAppSelect? datasetname $=9957$ ANX, last access: 15 July 2014.

National Research Council: Completing the Forecast: Characterizing and Communicating Uncertainty for Better Decisions Using Weather and Climate Forecasts, National Academies Press, Washingtion, D.C., available at: http://www.nap.edu/catalog. php?recordid=11699 (last access: 18 September 2014), 2006.
NCAR: Research Application Program, verication: Forecast Verication Utilities, http://CRAN.R-project.org/ (last access: September 2015), 2014.

NWS-OHD - National Weather Service, Office of Hydrologic Development: Ensemble Postprocessor (EnsPost) User's Manual, HEFS Release 0.3.2, available at: http://www.nws.noaa.gov/oh/ hrl/general/HEFS_doc/HEFS-0.3.2EnsPostUsers_Manual.pdf (last access: 22 July 2015), 2013.

Pielke, R. A.: Who Decides? Forecasts and Responsibilities in the 1997 Red River Flood, Appl. Behav. Sci. Rev., 7, 83-101, 1999.

Regonda, S. K., Seo, D.-J., Lawrence, B., Brown, J. D., and Demargne, J.: Short-term ensemble streamflow forecasting using operationally-produced single-valued streamflow forecasts - A Hydrologic Model Output Statistics (HMOS) approach, J. Hydrol., 497, 80-96, doi:10.1016/j.jhydrol.2013.05.028, 2013.

Seo, D.-J., Herr, H. D., and Schaake, J. C.: A statistical postprocessor for accounting of hydrologic uncertainty in short-range ensemble streamflow prediction, Hydrol. Earth Syst. Sci. Discuss., 3, 1987-2035, doi:10.5194/hessd-3-1987-2006, 2006.

Solomatine, D. P. and Shrestha, D. L.: A novel method to estimate model uncertainty using machine learning techniques, Water Resour. Res., 45, 1-16, doi:10.1029/2008WR006839, 2009.

US Department of Commerce, NOAA: NOAA/NWS Hydrologic Ensemble Forecasting, available at: http://www.nws.noaa.gov/ ohd/XEFS/ (last access: 22 July 2015), 2012.

USGS: Stream Site - USGS 05558300 Illinois River at Henry, IL, available at: http://waterdata.usgs.gov/nwis/inventory/?site_ no $=05558300 \&$ agencycd=USGS (last access: 2 February 2015), 2015a.

USGS: Stream Site - USGS 05587060 Illinois River at Hardin, IL, available at: http://waterdata.usgs.gov/il/nwis/inventory/?site_ no $=05587060 \&$ (last access: 3 February 2015), 2015 b.

Weerts, A. H., Winsemius, H. C., and Verkade, J. S.: Estimation of predictive hydrological uncertainty using quantile regression: examples from the National Flood Forecasting System (England and Wales), Hydrol. Earth Syst. Sci., 15, 255-265, doi:10.5194/hess-15-255-2011, 2011.

Welles, E., Sorooshian, S., Carter, G., and Olsen, B.: Hydrologic Verification: A Call for Action and Collaboration, B. Am. Meteorol. Soc., 88, 503-511, doi:10.1175/BAMS-88-4-503, 2007.

Wikipedia: Brier score, available from: http://en.wikipedia.org/ w/index.php?title=Brier_score\&oldid $=619686224$, last access: 27 August 2014

Wilson, L. J.: Verification of probability and ensemble forecasts, available at: http://www.swpc.noaa.gov/forecast_verification/ Assets/Tutorials/Ensemble_Forecast_Verification.pdf, last access: 27 August 2014.

Wood, A. W., Wiley, M., and Nijssen, B.: Use of quantile regression for calibration of hydrologic forecasts, available at: http: //ams.confex.com/ams/89annual/wrfredirect.cgi?id=10049 (last access: 2 February 2015), 2009.

WWRP/WGNE: Methods for probabilistic forecasts. Forecast Verification - Issues, Methods and FAQ, available at: http://www. cawcr.gov.au/projects/verification/verif_webpage.html\#BSS (last access: 27 August 2014), 2009. 